\title{
Benchmark Design and Prior-independent Optimization
}

\author{
Jason Hartline* $\quad$ Aleck Johnsen ${ }^{\dagger} \quad$ Yingkai Li ${ }^{\ddagger}$
}

September 9, 2020

\begin{abstract}
This paper compares two leading approaches for robust optimization in the models of online algorithms and mechanism design. Competitive analysis compares the performance of an online algorithm to an offline benchmark in worst-case over inputs, and prior-independent mechanism design compares the expected performance of a mechanism on an unknown distribution (of inputs, i.e., agent values) to the optimal mechanism for the distribution in worst case over distributions. For competitive analysis, a critical concern is the choice of benchmark. This paper gives a method for selecting a good benchmark. We show that optimal algorithm/mechanism for the optimal benchmark is equal to the prior-independent optimal algorithm/mechanism.

We solve a central open question in prior-independent mechanism design, namely we identify the prior-independent revenue-optimal mechanism for selling a single item to two agents with i.i.d. and regularly distributed values. We use this solution to solve the corresponding benchmark design problem. Via this solution and the above equivalence of prior-independent mechanism design and competitive analysis (a.k.a. prior-free mechanism design) we show that the standard method for lower bounds of prior-free mechanisms is not generally tight for the benchmark design program.
\end{abstract}

\footnotetext{
${ }^{*}$ Northwestern U., Evanston, IL. Email: hartline@u.northwestern.edu. Work done in part while supported by NSF CCF 1618502.

${ }^{\dagger}$ Northwestern U., Evanston IL. Email: aleckjohnsen@u.northwestern.edu. Work done in part while supported by NSF CCF 1618502.

${ }^{\ddagger}$ Northwestern U., Evanston IL. Email: yingkai.li@u.northwestern.edu Work done in part while supported by NSF CCF 1618502.
} 


\section{Introduction}

There are two leading approaches for robust optimization in the models of mechanism design and online algorithms (see detailed historical discussion in Section 6). A key property of these environments is that with the given constraints (incentive compatibility or online arrivals) the best outcome is unachievable pointwise. The first approach considers a benchmark and looks for a mechanism (resp. algorithm) that pointwise approximates the benchmark, a.k.a., prior-free approximation (resp. competitive analysis). The choice of benchmark is an important variable of this approach. The second approach assumes that the input is drawn at random from an unknown distribution in a family and looks for a mechanism that approximates, in worst-case over distributions in the family, the performance of the Bayesian optimal mechanism for the distribution, a.k.a., prior-independent approximation. (Respectively, this approach could also be applied to online algorithms.) This paper formalizes the problem of designing a good benchmark for priorfree approximation (resp. competitive analysis) and connects this benchmark design problem to prior-independent optimization.

Terminology and concrete examples from mechanism design are henceforth adopted for the majority of the paper. However, the main result relating benchmark design to prior-independent optimization does not rely on any specifics from mechanism design. Instead it applies to families of mechanisms represented by a family of functions from inputs to performances. In Section 5 we instantiate the framework for online learning and give results that parallel the specific developments for mechanism design.

The choice of benchmark in the first approach impacts the ability of approximation with respect to the benchmark to distinguish between good and bad mechanisms. On one hand a benchmark should be an upper bound on what is achievable by a mechanism; otherwise, approximating it does not necessarily mean that a mechanism is good. On the other hand it should not be too loose an upper bound; otherwise, neither good nor bad mechanisms can obtain good approximations and the degree to which we can distinguish good and bad mechanisms via the benchmark is limited.

As illustration, consider comparing the revenue of mechanisms for selling a digital good to $n$ agents with values $\boldsymbol{v}=\left(v_{1}, \ldots, v_{n}\right)$ bounded on $[1, H]$ to one of two benchmarks, the sum-of-values benchmark $\sum_{i} v_{i}$ and the price-posting-revenue benchmark $\max _{i} i v_{(i)}$ where $v_{(i)}$ is the $i$ th highest value. To ensure benchmarks give an upper bound on revenue, Hartline and Roughgarden (2008) suggested that the benchmark satisfies the property that, for any distribution on inputs from a given family of distributions, the expected benchmark (over the same distribution of inputs) be at least the expected performance of the optimal mechanism that knows the distribution. Thus approximation of the benchmark implies approximation of the optimal mechanism for any of the distributions in the family. We refer to this constraint as normalization. Both the sum-of-values and price-posting-revenue benchmarks are normalized (Hartline and Roughgarden, 2008).

Not all normalized benchmarks are equally good at discriminating between good and bad mechanisms. Consider the following two mechanisms. The random-sampling mechanism partitions the agents at random and offers the optimal price from each part to the other part (Goldberg et al., 2006). The random-power-pricing mechanism posts a take-it-or-leave-it price drawn from the uniform distribution on powers of two in $[1, H]$ (Goldberg and Hartline, 2003). These mechanisms and the benchmarks of the preceding paragraph are related as follows. On all inputs $\boldsymbol{v}$, the sum-of-values benchmark exceeds the random-power-pricing mechanism by a $\Theta(\log H)$ factor. On all inputs $\boldsymbol{v}$, the random sampling mechanism and the price-posting-revenue benchmark are $\Theta(1)$. Moreover, the performance of the latter benchmark and mechanism are always sandwiched between the former 
benchmark and mechanism and can equal either of them up to $\Theta(1)$. From this analysis, we see that the loose benchmark of sum-of-values does not discriminate between good mechanisms like random-sampling and bad mechanisms like random-power-pricing.

The preceding discussion suggests a benchmark design problem of identifying the normalized benchmark to which the tightest approximation is possible. We refer to the tightest approximation possible for a benchmark as its resolution (see Definition 3), as up to this factor the benchmark cannot distinguish between good and bad mechanisms. The sum-of-values benchmark has logarithmic resolution while the price-posting-revenue benchmark has constant resolution.

In summary: A family of distributions over inputs induces a class of normalized benchmarks. The benchmark admitting the tightest approximation, i.e. having smallest resolution, is optimal. The mechanism achieving this approximation is the prior-free optimal mechanism for the benchmark. Normalization of the benchmark implies that the prior-free approximation factor of a mechanism (to the benchmark) is at least its prior-independent approximation factor (for the normalizing family of distributions). A natural question is how this prior-free approach, and its optimal mechanism, compares to directly identifying the prior-independent optimal mechanism, i.e., the one with the best worst-case over distributions approximation to the Bayesian optimal mechanism.

The first main result of the paper is the general result that optimal benchmark design is equivalent to prior-independent optimization (Section 2). Described in the context of mechanism design, this result shows that the prior-free optimal mechanism for the optimal benchmark is the priorindependent optimal mechanism and that the optimal benchmark is simply the prior-independent optimal mechanism scaled up by its approximation factor (so as to satisfy the normalization constraint) 1 Consequently, it is not possible to identify optimal benchmarks and their corresponding optimal mechanisms for problems in which we are unable to solve the prior-independent optimization problem.

Our second main result is to solve the benchmark optimization problem (equivalently: solve the prior-independent mechanism design problem) for the problem of maximizing revenue from the sale of a single item to two agents for the family of i.i.d. regular value distributions (Section 33). This result answers a major question left open from Dhangwatnotai et al. (2015), Fu et al. (2015), and Allouah and Besbes (2018). The optimal mechanism is a mixture between the second-price auction, where each agent is offered a price equal to the highest of the other agents' values, and the auction where these prices are scaled up by a factor of about 2.5. Our solution to this central open question is the first example of a prior-independent optimal mechanism that arises as the solution to a non-trivial optimization problem and is not a standard mechanism from the literature (though the mechanism does fall into the lookahead family of mechanisms described by Ronen (2001) and has the same form as mechanisms used to prove bounds in Fu et al. (2015) and Allouah and Besbes (2018)). Our construction pins down the worst-case family of distributions. To solve the equivalent of benchmark design and prior-independent optimization, the approach of this paper is to directly solve the prior-independent optimization problem and use that solution to solve the benchmark design problem.

A key component of optimal benchmark design is tight lower bounds on the prior-free approximation of a benchmark. There is a standard method for lower bounding the approximation ratio

\footnotetext{
${ }^{1}$ The analysis that proves this equivalence is straightforward and, perhaps, obvious in hindsight. As we will describe below, there is an alternative benchmark design program which we view, in hindsight, as a relaxation of our benchmark design program. The prior literature strongly suggested that this alternative program and our program are equivalent; our third result shows that in fact the relaxation is lossy.
} 
of the best mechanism for a given benchmark (Goldberg et al., 2006). Consider the distribution over inputs for which all mechanisms achieve the same performance, e.g., for revenue maximization in mechanism design this distribution is the so-called equal-revenue distribution. The ratio of the expected value of the benchmark on this distribution to the revenue of any mechanism (all "undominated" mechanisms are the same) gives a lower bound on the approximation factor of any mechanism to the benchmark. Hartline and McGrew (2005) proved that this approach is tight for a large family of benchmarks and $n=3$ agents. Chen et al. (2014) proved that this approach is tight for a large family of benchmarks and a general number $n$ of agents. Thus, a natural approach to solve the benchmark optimization problem is to relax the program to optimize, not the approximation factor of the best mechanism, but the lower bound on the best approximation factor from the above approach. Our third main result - without being able to explicitly solve this relaxed program - is that the relaxation is not without loss, i.e., it gives a normalized benchmark for which the lower bound is not achievable by any mechanism (Section 44).

Our proof of the third main result follows from the second main result and a counter example that shows that there is a benchmark that achieves a lower objective value (for the relaxed benchmark program) than the approximation ratio of the optimal prior-independent auction (which our first result shows to be the optimal objective value of the original benchmark program).

Our second and third results are proved under the restriction of mechanisms that are (a) dominant strategy incentive compatible (DSIC), i.e., where truthtelling is a good strategy for each agent regardless of the strategies of other agents, and (b) scale invariant. It is known that there are environments for prior-independent mechanism design where DSIC is not without loss (Feng and Hartline, 2018). The restricted family of DSIC mechanisms is interesting even if it is with loss; however, far more study of non-incentive-compatible mechanisms is warranted. It is not known whether scale invariance is without loss or not, though Allouah and Besbes (2018) conjecture that it is without loss. This question is important and remains open.

There is an important negative interpretation of our first result, that the prior-free benchmark optimization problem and the prior-independent optimization problem give the same answer (which has consequences for both mechanism design and online algorithms). One reason to prefer priorfree analysis over prior-independent analysis is that it could be more robust. Of course to be more robust, as Hartline and Roughgarden (2008) have recommended, we need to choose a normalized benchmark, i.e., one for which prior-free approximation implies prior-independent approximation. With many possible normalized benchmarks, we need a method for selecting one. We have adopted a natural formal method for selecting one, namely, the one that admits the tightest approximation is the best one. However, the results of the paper show that the answer we will then get from studying prior-free approximation of this optimal benchmark is the same as the answer we will get from the original prior-independent question. Thus, there is no added robustness from the prior-free approximation of the optimal benchmark (over prior-independent analysis). Of course, there are environments where increased robustness can informally be observed from prior-free approximation of ad hoc, i.e., non-optimal, benchmarks. This observations suggests the main open question of this paper which is to identify a rigorous framework for evaluating prior-free benchmarks which leads to an additional desired robustness over the prior-independent framework. We will formally describe this shortcoming of the framework with the example environment of no-regret online learning in Section 5 , 


\section{Benchmark Optimization Is Prior-independent Optimization}

We formulate the benchmark optimization problem in abstract terms and prove that it is equivalent to prior-independent optimization. Our framework and results in this section hold generally for algorithm design, however, we will adopt notation and terminology for mechanism design to maintain consistency with the discussion in subsequent sections. Notably, the development of this section makes no assumptions on the families of distributions over the input that are considered.

Denote the space of inputs by $\mathcal{V}$ and an input in this space by $\boldsymbol{v}$. Denote a family of distributions over input space by $\mathcal{F} \subset \Delta(\mathcal{V})$ and a distribution in the family by $F$. Denote a family of feasible mechanisms by $\mathcal{M}$ and a mechanism in this family by $M$. Denote a family of benchmarks by $\mathcal{B}$ and a benchmark in this family by $B$. For our purposes we will view both a mechanism and a benchmark as a function that maps the input space to an expected performance (e.g., in the case the mechanism is randomized), denoted respectively by $M(\boldsymbol{v})$ and $B(\boldsymbol{v})$. When evaluating the performance of a mechanism or benchmark in expectation over the distribution we adopt the short-hand notation $M(F)=\mathbf{E}_{\boldsymbol{v} \sim F}[M(\boldsymbol{v})]$ and $B(F)=\mathbf{E}_{\boldsymbol{v} \sim F}[B(\boldsymbol{v})]$.

In these abstract terms we formally define the Bayesian, prior-independent, and prior-free optimization problems.

Definition 1. The Bayesian optimal mechanism design problem is given by a distribution $F$ and family of mechanisms $\mathcal{M}$ and asks for the mechanism $\mathrm{OPT}_{F}$ with the maximum expected performance:

$$
\mathrm{OPT}_{F}=\underset{M \in \mathcal{M}}{\operatorname{argmax}} M(F)
$$

Definition 2. The prior-independent mechanism design problem is given by a family of mechanisms $\mathcal{M}$ and a family of distributions $\mathcal{F}$ and solves the program

$$
\beta=\min _{M \in \mathcal{M}} \max _{F \in \mathcal{F}} \frac{\operatorname{OPT}_{F}(F)}{M(F)} .
$$

Next, $\rho^{B}$ is the approximation ratio of the optimal prior-free mechanism for benchmark $B$; in the subsequent discussion of benchmark design, we reinterpret $\rho^{B}$ as the resolution of benchmark $B$.

Definition 3. The prior-free mechanism design problem is given by a family of mechanisms $\mathcal{M}$ and $a$ benchmark $B$ and solves the program

$$
\rho^{B}=\min _{M \in \mathcal{M}} \max _{\boldsymbol{v} \in \mathcal{V}} \frac{B(\boldsymbol{v})}{M(\boldsymbol{v})} .
$$

Recall from the introduction, benchmarks with small resolution are better at differentiating good mechanisms from bad one. Both prior-independent and prior-free mechanism design problems are searching for mechanisms with robust performance guarantees. In principle, prior-free guarantees can provide more robustness than prior-independent guarantees as the guarantee is required to hold pointwise on all inputs rather than in expectation according to the distribution. Whether or not a prior-free guarantee is meaningful depends on the choice of benchmark. The possibility that some benchmarks might be better than others for meaningfully quantifying the performance of a mechanism suggests that benchmarks themselves can be optimized. 
Hartline and Roughgarden (2008) recommend restricting attention to benchmarks that satisfy the following normalization property which requires that the benchmark is an upper bound on the optimal performance of a mechanism. Rather than measure this optimal performance pointwise as is common with online algorithms, Hartline and Roughgarden (2008) recommend measuring this optimal performance in expectation with respect to any distribution in a family of distributions. This way of measuring the optimal performance can take into account the constraints on the mechanism, i.e., that $M \in \mathcal{M} \mathbb{2}$ The normalization constraint implies a strong guarantee: A mechanism that is a $\rho$ approximation to a normalized benchmark guarantees a $\rho$ prior-independent approximation.

Definition 4. A benchmark $B$ is normalized for a family of distributions $\mathcal{F}$ and family of mechanisms if for every distribution in the family the expected benchmark is at least the optimal expected performance, i.e.,

$$
B(F) \geq \mathrm{OPT}_{F}(F), \quad \forall F \in \mathcal{F} .
$$

Denote the normalized benchmarks for $\mathcal{F}$ by $\mathcal{B}(\mathcal{F})$.

Proposition 1 (Hartline and Roughgarden, 2008). If mechanism $M$ is a prior-free $\rho$ approximation of a benchmark $B$ normalized to distributions $\mathcal{F}$ then its prior-independent approximation for distributions $\mathcal{F}$ is at most $\rho$.

The point of mechanism design is a principled method for choosing one mechanism over another. The prior-free framework described above gives such a method only in so far as approximation of the benchmark distinguished between good mechanisms and bad ones. For example, no mechanism will approximate a prior-free benchmark that is too large, thus, good mechanisms will not necessarily be separated from bad ones. Recall the discussion of the sum-of-values and postedprice-revenue benchmarks in the introduction. One way to quantify the inability of a benchmark to discriminate is by considering the approximation factor of the best mechanisms for the benchmark, i.e., the benchmark's resolution (Definition 3). Our benchmark design program aims to identify the benchmark with the finest resolution.

Definition 5. The benchmark design problem for family of distributions $\mathcal{F}$, family of mechanisms $\mathcal{M}$, and space of inputs $\mathcal{V}$ solves the program

$$
\gamma=\min _{B \in \mathcal{B}(\mathcal{F})} \rho^{B}=\min _{B \in \mathcal{B}(\mathcal{F})} \min _{M \in \mathcal{M}} \max _{\boldsymbol{v} \in \mathcal{V}} \frac{B(\boldsymbol{v})}{M(\boldsymbol{v})} .
$$

We are now ready to state and prove the main result of this section, that the benchmark design problem and the prior-independent mechanism design problem are equivalent. Before doing so it should be noted that it is not generally understood how to solve these problems. (We will, however, give a solution to a paradigmatic prior-independent mechanism design problem in the next section.)

Theorem 1. For any family of distributions $\mathcal{F}$, any set of mechanisms $\mathcal{M}$, benchmark design is equivalent to prior-independent mechanism design, i.e., $\gamma=\beta$, and the optimal benchmark is given by the performance of the prior-independent optimal mechanism scaled up by its approximation ratio $\beta$.

\footnotetext{
${ }^{2}$ For mechanism design, these constraints will be incentive compatibility and individual rationality. For online algorithms these constraints are that the current decision must be made before the future input is known.
} 
This theorem follows from a corollary of Proposition 1 which shows that $\beta \leq \gamma$ and the following lemma which shows that $\gamma \leq \beta$.

Corollary 1. For any families of distributions and mechanisms, the prior-independent optimal ratio $\beta$ is at most the optimal benchmark ratio $\gamma$, i.e., $\beta \leq \gamma$.

Proof. By the definition of program $(\sqrt[\gamma]{ })$, the optimal mechanism for the optimal benchmark is a prior-free $\gamma$ approximation. By Proposition 1 and the normalization of the optimal benchmark, this mechanism is a prior-independent $\gamma$ approximation. The optimal prior-independent mechanism is no worse, i.e., $\beta \leq \gamma$.

Lemma 1. For any families of distributions and mechanisms, the optimal benchmark ratio $\gamma$ is at most the prior-independent optimal ratio $\beta$, i.e., $\gamma \leq \beta$.

Proof. Consider the prior-independent optimal mechanism $M^{*}$ with approximation $\beta$. Define the benchmark

$$
B^{*}(\boldsymbol{v})=\beta M^{*}(\boldsymbol{v}),
$$

i.e., the benchmark is the performance of the prior-independent optimal mechanism scaled up by its approximation factor. Taking the expectation of $\boldsymbol{v}$ drawn from any distribution $F$, we have

$$
B^{*}(F)=\beta M^{*}(F) .
$$

First, notice that $B^{*}$ is normalized. Since $M^{*}$ is a prior-independent $\beta$-approximation, $M^{*}(F) \geq$ $\frac{1}{\beta} \operatorname{OPT}_{F}(F)$ for all $F$ in the family of distributions. Multiplying through by $\beta$ and applying equation (2) shows that the benchmark meets the definition of normalization.

Second, equation (11) implies that $M^{*}$ is a prior-free $\beta$-approximation of $B^{*}$. Thus, $\left(M^{*}, B^{*}\right)$ is a solution to the benchmark design program $(\sqrt{\gamma})$ with ratio $\beta$. The optimal solution to the program is no larger. Thus, $\gamma \leq \beta$.

The benchmark design problem presented above asks for both an optimal benchmark and the optimal mechanism for this benchmark. Fixing the benchmark, the problem of identifying the optimal mechanism is not well understood. There is a canonical method for identifying lower bounds on the approximation ratio of the optimal mechanism. Goldberg et al. (2006) suggest that the prior-free approximation any mechanism for a benchmark can be lower bounded by identifying a distribution over inputs for which all non-dominated mechanisms obtain the same performance. For revenue maximizing mechanism design, this distribution is the so-called equal revenue distribution. The following lemma and definition generalize this lower bound to environments where a mechanism neutralizing distribution may not exist.

Lemma 2. For any benchmark $B$, distribution $F$, and family of mechanisms which induce $\mathrm{OPT}_{F}$, the optimal prior-free approximation $\rho^{M}$ is at least $\frac{B(F)}{\mathrm{OPT}_{F}(F)}$.

Proof. Let $M^{B}$ be the prior-free optimal mechanism for benchmark $B$, i.e., that optimizes the program $\rho^{B}$, then

$$
\rho^{B}=\max _{\boldsymbol{v} \in \mathcal{V}} \frac{B(\boldsymbol{v})}{M^{B}(\boldsymbol{v})} \geq \frac{B(F)}{M^{B}(F)} \geq \frac{B(F)}{\mathrm{OPT}_{F}(F)}
$$


Definition 6. For any benchmark $B$, family of distributions $\mathcal{F}$, and family of mechanisms which induce $\mathrm{OPT}_{F}$, the canonical lower-bound on the optimal prior-free approximation of $B$ is

$$
\bar{\rho}^{B}=\max _{F \in \mathcal{F}} \frac{B(F)}{\operatorname{OPT}_{F}(F)} .
$$

This lower bound holds for any family of distributions; e.g., contrasting to standard assumptions in mechanism design, it allows distributions that are irregular and correlated. Moreover, the lower bound is tight for a number of interesting benchmarks. By Theorem 1, the optimal benchmark $B^{*}$ satisfies $\rho^{B^{*}}=\bar{\rho}^{B^{*}}$. For the digital goods revenue maximization problem, a large family of benchmarks were shown by Chen et al. (2014) to also satisfy this equality. Thus, a natural relaxation of the benchmark design program is, instead of optimizing benchmarks that admit the best prior-free approximation, to optimize benchmarks that admit the best lower bound of Definition 6 .

Definition 7. The relaxed benchmark design problem for distribution family $\mathcal{F}$ and family of mechanisms that defines the Bayesian optimal mechanism $\mathrm{OPT}_{F}$ for and distribution $F \in \mathcal{F}$ solves the program:

$$
\bar{\gamma}=\min _{B \in \mathcal{B}(\mathcal{F})} \max _{F \in \mathcal{F}} \frac{B(F)}{\operatorname{OPT}_{F}(F)}
$$

Proposition 2. The value of the relaxed program lower bounds optimal resolution, i.e., $\bar{\gamma} \leq \gamma$.

We will see in Section 4 that the relaxation is not generally without loss and, in particular, the optimal benchmark for the relaxed program can have $\bar{\gamma}<\gamma$. We will show the strictness of this inequality by example for the paradigmatic problem of maximizing revenue from the sale of an item to one of two agents and benchmarks that are normalized for value distributions that are i.i.d. and regular. We show this inequality is strict for online learning as well.

\section{Prior-Independent Optimal Mechanisms}

In this section we consider the problem of maximizing revenue from the sale of a single item to one of two agents with values distributed independently and identically from a distribution that satisfies a natural convexity property (to be defined formally). We identify the prior-independent optimal mechanism and thus, by the equivalence between benchmark design and prior-independent optimization, the optimal benchmark. The section begins with preliminary discussion of mechanism design.

\subsection{Mechanism Design Preliminaries}

Consider $n$ agents with private values $\boldsymbol{v}=\left(v_{1}, \ldots, v_{n}\right)$. The agents have linear utility given, e.g, agent $i$ 's utility is $v_{i} x_{i}-p_{i}$ for allocation probability $x_{i}$ and expected payment $p_{i}$. Agents' values are drawn independently and identically from a product distribution $\boldsymbol{F}=F \times \cdots \times F$ where $F$ will denote the cumulative distribution function of each agent's value.

A mechanism $M$ is defined by an ex post allocation and payment rule $\boldsymbol{x}^{M}$ and $\boldsymbol{p}^{M}$ which map the profile of values $\boldsymbol{v}$ to a profile of allocation probabilities and a profile of payments, respectively. We focus on mechanisms that are feasible, dominant strategy incentive compatible, and individually rational: 
- For selling a single item, a mechanism is feasible if for all valuation profiles, the allocation probabilities sum to at most one, i.e., $\forall \boldsymbol{v}, \sum_{i} x_{i}^{M}(\boldsymbol{v}) \leq 1$.

- A mechanism is dominant strategy incentive compatible if no agent $i$ with value $v_{i}$ prefers to misreport some value $z: \forall \boldsymbol{v}, i, z, v_{i} x_{i}^{M}(\boldsymbol{v})-p_{i}^{M}(\boldsymbol{v}) \geq v_{i} x_{i}^{M}\left(z, \boldsymbol{v}_{-i}\right)-p_{i}^{M}\left(z, \boldsymbol{v}_{-i}\right)$ where $\left(z, \boldsymbol{v}_{-i}\right)$ denotes the valuation profile with $v_{i}$ replaced with $z$.

- A mechanism is individually rational if truthful reporting always leads to non-negative utility: $\forall \boldsymbol{v}, i, v_{i} x_{i}^{M}(\boldsymbol{v})-p_{i}^{M}(\boldsymbol{v}) \geq 0$.

A mechanism's revenue can be easily and geometrically understood via the marginal revenue approach of Myerson (1981) and Bulow and Roberts (1989). For distribution $F$, the quantile $q$ of an agent with value value $v$ denotes how strong that agent is relative to the distribution $F$. Quantiles are defined by the mapping $Q_{F}(v)=1-F(v)$. Denote the mapping back to value space by $V_{F}$, i.e., $V_{F}(q)$ is the value of the agent with quantile $q$. A single agent price-posting revenue curve gives the revenue of posting a price as a function of the probability that the agent accepts the price. For an agent with value distribution $F$, price $V_{F}(q)=F^{-1}(1-q)$ is accepted with probability $q$; its revenue is $q V_{F}(q)$. A single agent revenue curve gives the optimal revenue from selling to a single agent $R_{F}(q)$ as a function of ex ante sale probability $q$. Note that the revenue curve $R$ is always concave. The agent is regular if her revenue curve equals her price-posting revenue curve. The optimal mechanism for a single agent posts the monopoly price $V_{F}\left(\hat{q}^{\star}\right)$ which corresponds to the monopoly quantile $\hat{q}^{\star}=\operatorname{argmax}_{q} R_{F}(q)$. The expected revenue of a multi-agent mechanism $M$ is equal to its surplus of marginal revenue.

Theorem 2 (Myerson, 1981). Given any incentive-compatible mechanism $M$ with allocation rule $\boldsymbol{x}^{M}(\boldsymbol{v})$, the expected revenue of mechanism $M$ for agents with regular distribution $\boldsymbol{F}$ is equal to its expected surplus of marginal revenue, i.e.,

$$
M(\boldsymbol{F})=\sum_{i} \mathbf{E}_{\boldsymbol{v} \sim \boldsymbol{F}}\left[p_{i}^{M}(\boldsymbol{v})\right]=\sum_{i} \mathbf{E}_{\boldsymbol{v} \sim \boldsymbol{F}}\left[R_{F}^{\prime}\left(Q_{F}\left(v_{i}\right)\right) x_{i}^{M}(\boldsymbol{v})\right] .
$$

Corollary 2 (Myerson, 1981). For i.i.d., regular, single-item environments, the optimal mechanism $\mathrm{OPT}_{F}$ is the second-price auction with reserve equal to the monopoly price.

Proof. Optimizing marginal revenue pointwise the item is assigned to the agent with the highest non-negative marginal revenue. Since agents are i.i.d. and the marginal revenue curves are monotonically non-increasing, this winning agent is the one with the highest value that exceeds the monopoly price.

The following lemma from Dhangwatnotai et al. (2015) follows from Theorem 2 and gives a geometric understanding of revenue in two-agent auctions.

Lemma 3 (Dhangwatnotai et al., 2015). In i.i.d. two-agent single-item environments, the expected revenue of the second price auction is twice the area under the revenue curve and the expected revenue of the optimal mechanism is twice the area under the smallest monotone concave upper bound of the revenue curve. 


\subsection{Prior-independent Optimization}

In the remainder of this section we solve for the prior-independent optimal mechanism for the revenue objective with the restriction to

- single-item, two-agent environments, i.e., $n=2$ (implicit);

- the family of i.i.d. regular value distribution $\mathcal{F}^{\text {Reg}}$; and

- the family of feasible, incentive compatible, individually rational, and scale-invariant mechanisms $\mathcal{M}^{\mathrm{SI}}$.

The following discussion motivates these restrictions. The single-item two-agent environment is canonical for prior-independent revenue maximization. There do not exist good prior-independent mechanisms for general asymmetric and irregularly distributed agent values. Almost all papers on prior-independent mechanism design restrict to i.i.d. agents. Almost all papers on revenue maximization for prior-independent mechanism design restrict to regular distributions. The restriction to feasible and individually rational mechanisms is required to have a sensible optimization problem. The restriction to incentive compatible mechanisms is made in almost all papers on prior-independent mechanism design, an exception is Feng and Hartline (2018) where it is shown that the restriction can be lossy. The remaining condition which we formally define below is scale invariance.

Definition 8. Given any incentive-compatible mechanism $M$ with allocation rule $x^{M}(\boldsymbol{v})$, mechanism $M$ is scale invariant if for each agent $i$, valuation profile $\boldsymbol{v}$ and any constant $\alpha>0$, $x_{i}^{M}(\alpha \cdot \boldsymbol{v})=x_{i}^{M}(\boldsymbol{v})$. Scale invariance further implies $M(a \cdot \boldsymbol{v})=a \cdot M(\boldsymbol{v})$.

Allouah and Besbes (2018) prove that the optimal prior-independent mechanism among a broad family of mechanisms is scale invariant. They show that if $\lim _{\alpha \rightarrow 0} x_{i}(\alpha \cdot \boldsymbol{v})$ always exists for mechanisms in the family, then the optimal prior-independent mechanism is scale invariant. They conjecture that this weaker assumption is without loss; if true, the mechanism we identify as the optimal mechanism among scale-invariant mechanisms is also prior-independent optimal among all mechanisms.

Given the restriction to scale-invariant mechanisms, it will be sufficient to consider distributions that are normalized so that the single-agent optimal revenue is $\max _{q} R(q)=1$.

The following family of (stochastic) markup mechanisms is (essentially, in $n=2$ agent environments) the restriction of the family of lookahead mechanisms (Ronen, 2001) to those that are scale invariant. Notice that the second-price auction is the 1-markup mechanism $M_{1}$.

Definition 9. The $r$-markup mechanism $M_{r}$ identifies the agent with the highest-value (and ties broken uniformly at random) and offers this agent $r$ times the second-highest value. A stochastic markup mechanism draws $r$ from a given distribution on $[1, \infty)$. The family of stochastic markup mechanisms is $\mathcal{M}^{\text {SMKUP }}$.

Theorem 3. For i.i.d., regular, two-agent, single-item environments, the optimal scale-invariant, incentive-compatible mechanism for prior-independent optimization program (B) is $M_{\alpha^{*}, r^{*}}$ which randomizes over the second-price auction $M_{1}$ with probability $\alpha^{*}$ and $r^{*}$-markup mechanism $M_{r^{*}}$ with probability $1-\alpha^{*}$, where $\alpha^{*} \approx 0.806$ and $r^{*} \approx 2.447$. The worst-case regular distribution for this mechanism is triangle distribution $\operatorname{Tri}_{\bar{q}^{*}}$ with $\bar{q}^{*} \approx 0.093$ and its approximation ratio is $\beta \approx 1.907$. 
In the two sections below we prove this theorem with the following main steps. First, we characterize the prior-independent optimal mechanism under the restriction to stochastic markup mechanisms and triangle distributions, cf. Alaei et al. (2018). This restricted program has the same solution as is given in Theorem 3. Second we show that the stochastic markup mechanisms and triangle distributions are mutual best responses among the more general families of scale-invariant mechanisms and regular distributions. Combining these results gives the theorem.

\subsection{Stochastic Markup Mechanisms versus Triangle Distributions}

In this section we characterize the solution to the prior-independent optimization program restricted to stochastic markup mechanisms and triangle distributions. We first define triangle distributions, which have revenue curves shaped like triangles (Figure 1), as well as a more general family of truncated distributions, which will be important subsequently in the proof. Recall that for scaleinvariant mechanisms, it is without loss to normalize the distributions to have monopoly revenue one.

Definition 10. A normalized triangle distribution with monopoly quantile $\bar{q}$, denoted $\operatorname{Tri}_{\bar{q}}$, is defined by the quantile function

$$
Q_{\operatorname{Tri}_{\bar{q}}}(v)= \begin{cases}\frac{1}{1+v(1-\bar{q})} & v \leq 1 / \bar{q} \\ 0 & \text { otherwise. }\end{cases}
$$

The triangulation of a normalized distribution with monopoly quantile $\bar{q}$ is $\operatorname{Tri}_{\bar{q}}$. The family of normalized triangle distributions is $\mathcal{F}^{\operatorname{Tri}}=\left\{\operatorname{Tri}_{\bar{q}}: \bar{q} \in[0,1]\right\}$.

Definition 11. A distribution is truncated if the highest-point in its support is the monopoly price (typically a point mass). The truncation of a distribution is the distribution that replaces every point above the monopoly price with the monopoly price. The family of truncated distributions is denoted $\mathcal{F}^{\text {Trunc }}$.

The three lemmas below give formulae for the revenue of the optimal mechanism, the secondprice auction, and non-trivial markup mechanisms for triangle distributions. The formula for revenue of markup mechanisms is discontinuous at $r=1$. Thus, in our discussion we will distinguish between the second-price auction $M_{1}$ and non-trivial markup mechanism $M_{r}$ for $r>1$.

Lemma 4. For i.i.d., normalized truncated, two-agent, single-item environments, the optimal mechanism posts the monopoly price and obtains revenue $2-\bar{q}$ where $\bar{q}$ is the probability that an agent's value equals the monopoly price.

Proof. The smallest monotone concave function that upper bounds the revenue curve is a trapezoid; its area is $\bar{q} / 2+1-\bar{q}$. The optimal revenue from two agents, by Lemma 3 , is twice this area, i.e., $2-\bar{q}$.

Lemma 5. The revenue of the second-price auction $M_{1}$ for distribution $\operatorname{Tr}_{\bar{q}}$ is 1, i.e., $M_{1}\left(\operatorname{Tr}_{\bar{q}}\right)=1$.

Proof. By Lemma 3, the revenue is twice the area under the revenue curve. That area is $1 / 2$; thus, the revenue is 1 . 

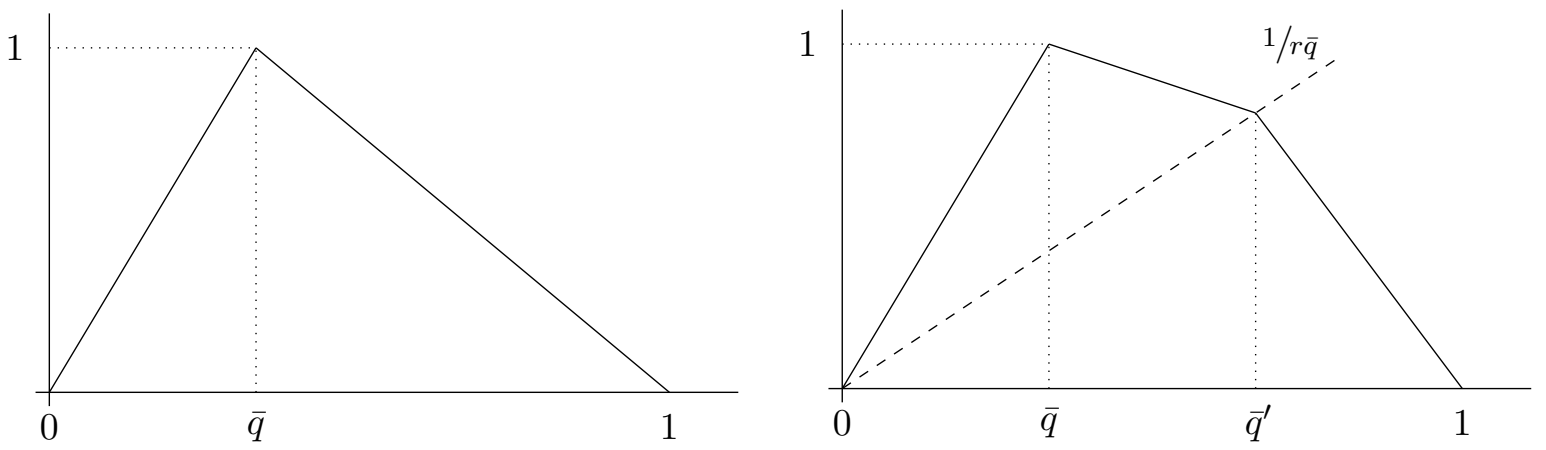

Figure 1: The left hand side is the revenue curve for triangle distribution $\operatorname{Tri}_{\bar{q}}$ and the right hand side is the revenue curve for quadrilateral distribution $\mathrm{Qr}_{\bar{q}, \bar{q}^{\prime}, r}$. The definition of quadrilateral distribution $\mathrm{Qr}_{\bar{q}, \bar{q}^{\prime}, r}$ will be formally introduced later in Section 3.4.

Lemma 6. The revenue of the $r$-markup mechanisms $M_{r}$ on triangle distribution $\operatorname{Tri}_{\bar{q}}$, for $r \in$ $(1, \infty)$ and $\bar{q} \in[0,1)$, is

$$
M_{r}\left(\operatorname{Tri}_{\bar{q}}\right)=\frac{2 r}{(1-\bar{q})(r-1)}\left(\frac{1-\bar{q}}{1-\bar{q}+\bar{q} r}+\frac{\ln \left(\frac{r}{1-\bar{q}+\bar{q} r}\right)}{1-r}\right) .
$$

The proof of Lemma 6 is straightforward and given in Appendix $\mathrm{A}$.

The following theorem characterizes the prior-independent optimal stochastic markup mechanism against triangle distributions. The parameters of this optimal mechanism are the solution to an algebraic expression (cf. Lemma [6) that we are unable to solve analytically. Our proof will instead combine numeric calculations of select points in parameter space with theoretical analysis to rule out most of the parameter space. For the remaining parameter space, we can show that the expression is well-behaved and, thus, numeric calculation can identify near optimal parameters. Discussion of this hybrid numerical and theoretical analysis can be found in Appendix $\mathrm{A}$.

Before giving Theorem 4 , we give context for its proof. In abstract terms, the prior-independent optimization program $(\beta)$ can be viewed as a zero sum game between the designer and an adversary, where the designer chooses a prior-independent mechanism $M$, the adversary chooses a worst-case distribution $F$ (and its induced revenue curve), and the payoff of the designer is the approximation ratio $\operatorname{OPT}_{F}(F) / M(F)$ (see Definition 2).

Theorem 4. For i.i.d., triangle distribution, two-agent, single-item environments, the optimal stochastic markup mechanism for prior-independent optimization program (B) is $M_{\alpha^{*}, r^{*}}$ which randomizes over the second-price auction $M_{1}$ with probability $\alpha^{*}$ and $r^{*}$-markup mechanism $M_{r^{*}}$ with probability $1-\alpha^{*}$, where $\alpha^{*} \approx 0.806$ and $r^{*} \approx 2.447$. The worst-case distribution for this mechanism is the triangle distribution $\operatorname{Tri}_{\bar{q}^{*}}$ with $\bar{q}^{*} \approx 0.093$ and its approximation ratio is $\beta \approx 1.907$.

Proof. The approach of this proof is to identify the triangle Tri $\bar{q}_{\bar{q}^{*}}$ for which the designer is indifferent between the second price auction $M_{1}$ and the optimal (non-trivial) markup mechanism, denoted $M_{r^{*}}$. For such a distribution $\operatorname{Tri}_{\bar{q}^{*}}$, the designer is also indifferent (in minimizing the approximation ratio) between any mixture over $M_{1}$ (with probability $\alpha$ ) and $M_{r^{*}}$ (with probability $1-\alpha$ ), and all other $r$-markup mechanisms for $r \notin\left\{1, r^{*}\right\}$ are inferior (Figure 21). We then identify the $\alpha^{*}$ 

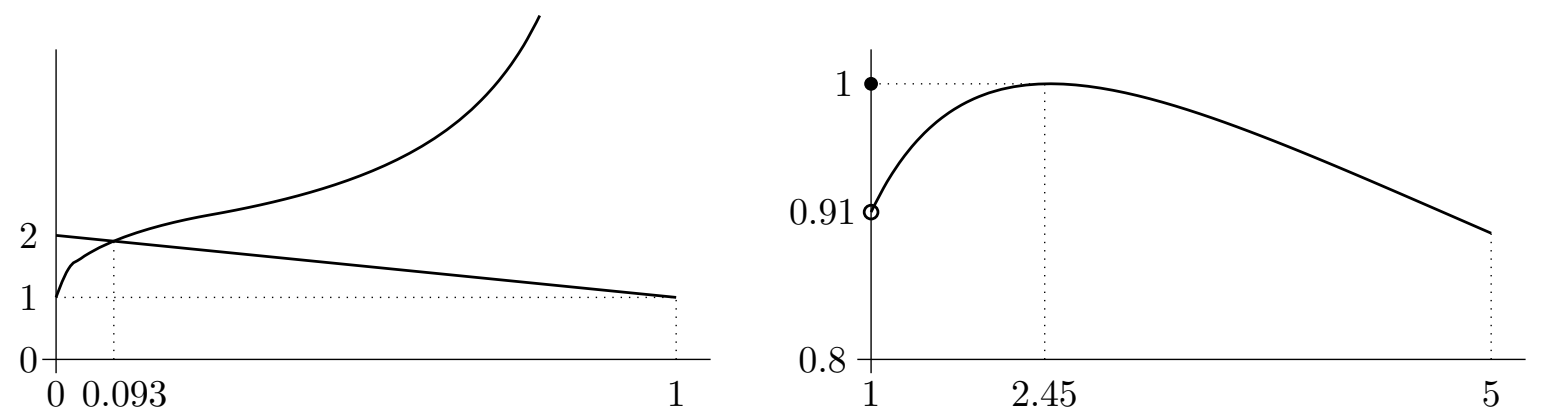

Figure 2: The figure on the left plots, as a function of $\bar{q}$, the approximation ratio $\operatorname{APX}_{1}(\bar{q})$ of the second-price auction $M_{1}$ against triangle distribution $\operatorname{Tri}_{\bar{q}}$ (straight line), and the approximation ratio $\operatorname{APX}_{*}(\bar{q})$ of the optimal non-trivial markup mechanism against triangle distribution $\operatorname{Tri}_{\bar{q}}$ (curved line). These functions cross at $\bar{q}^{*}=0.0931057$. The figure on the right plots the revenue of the $r$ markup mechanism $M_{r}$ on triangle distribution Tri $\bar{q}_{\bar{q}^{*}}$ as a function of markup $r$, i.e., $M_{r}\left(\operatorname{Tri}_{\bar{q}^{*}}\right)$. Notice that, by choice of $\bar{q}^{*}$, the optimal non-trivial markup mechanism has the same revenue as the second-price auction.

for which the adversary's best response (in maximizing the approximation ratio) to $M_{\alpha^{*}, r^{*}}$ is the distribution $\operatorname{Tri}_{\bar{q}^{*}}$. This solution $M_{\alpha^{*}, r^{*}}$ and $\operatorname{Tri}_{\bar{q}^{*}}$ is a Nash equilibrium between the designer and adversary and, thus, it solves the prior-independent optimization problem. The parameters can be numerically identified as $\alpha^{*} \approx 0.80564048, r^{*} \approx 2.4469452, \bar{q}^{*} \approx 0.0931057$, and the approximation ratio is $\beta \approx 1.9068943$.

First, we identify the triangle distribution $\bar{q}^{*}$ and the $r^{*}$ for which $M_{1}$ and $M_{r^{*}}$ obtain the same ratio. Denote the approximation ratio for the second-price auction $M_{1}$ as $\operatorname{APX}_{1}(\bar{q})=2-\bar{q}$ (the ratio of Lemma 4 to Lemma 5 ), which is continuous in $\bar{q}$. Denote the approximation ratio of the optimal markup mechanism against distribution $\operatorname{Tri}_{\bar{q}}$ by $\operatorname{APX}_{*}(\bar{q})=\sup _{r>1} \frac{\operatorname{OPT}_{\operatorname{Tr} i \bar{q}}\left(\operatorname{Tri}_{\bar{q}}\right)}{M_{r}\left(\operatorname{Tri}_{\bar{q}}\right)}$. By Lemma 6, the approximation ratio $\operatorname{APX}_{*}(\bar{q})$ is continuous in $\bar{q}$ as well. It is easy to verify that $\operatorname{APX}_{1}(0)=2>\operatorname{APX}_{*}(0)=1$ while $\operatorname{APX}_{1}(1)=1<\operatorname{APX}_{*}(1)=\infty$. By continuity, there exists a $\bar{q}^{*}$ where these two functions cross, i.e., $\operatorname{APX}_{*}\left(\bar{q}^{*}\right)=\operatorname{APX}_{1}\left(\bar{q}^{*}\right)$. See Figure 2. By numerical calculation, $\bar{q}^{*} \approx 0.0931057$, and

$$
r^{*}=\underset{r>1}{\operatorname{argmax}} \frac{\mathrm{OPT}_{\operatorname{Tri}_{\bar{q}^{*}}}\left(\operatorname{Tri}_{\bar{q}^{*}}\right)}{M_{r}\left(\operatorname{Tri}_{\bar{q}^{*}}\right)} \approx 2.4469452 .
$$

Now, fixing $r^{*}$, we search for $\alpha^{*}$ for which the adversary maximizes the approximation ratio of mechanism $M_{\alpha^{*}, r^{*}}$ by selecting triangle distribution $\operatorname{Tri}_{\bar{q}^{*}}$. Denote by $\bar{q}_{r}(\alpha)$ the monopoly quantile as a function of $\alpha$ for the triangle distribution that maximizes the approximation ratio of mechanism $M_{\alpha, r}$, i.e.,

$$
\bar{q}_{r}(\alpha)=\underset{\bar{q}}{\operatorname{argmax}} \frac{\mathrm{OPT}_{\operatorname{Tri}_{\bar{q}}}\left(\operatorname{Tri}_{\bar{q}}\right)}{M_{\alpha, r}\left(\operatorname{Tri}_{\bar{q}}\right)} .
$$

By numerical calculation, for any $r \in[2.445,2.449], \bar{q}_{r}(0.81)<\bar{q}^{*}<\bar{q}_{r}(0.8)$. Continuity of $\bar{q}_{r}(\cdot)$ for $r \in[2.445,2.449]$ and $\alpha \in[0.8,0.81]$ (formally proved in Appendix $\mathrm{A}$ ), then implies that there exists $\alpha^{*}$ such that $\bar{q}_{r^{*}}\left(\alpha^{*}\right)=\bar{q}^{*}$. By numerical calculation, $\alpha^{*} \approx 0.80564048$. 


\subsection{Mutual best-response of Stochastic Markup Mechanisms and Triangle Dis- tributions}

In this section we show that stochastic markup mechanisms are a best response (for the designer) to truncated distributions and that truncated distributions are a best response (for the adversary) to stochastic markup mechanisms. Moreover, we show that among truncated distributions, triangle distributions are the best for the adversary. Triangle distributions are known to be worst case for other questions of interest in mechanism design, e.g., approximation by anonymous reserves and anonymous pricings (Alaei et al., 2018). The proof that triangle distributions are worst-case for two-agent prior-independent revenue maximization is significantly more involved than these previous results.

Theorem 5. For i.i.d., two-agent, single-item environments and any scale-invariant incentivecompatible mechanism $M$, there is a stochastic markup mechanism $M^{\prime}$ with (weakly) higher revenue (and weakly lower approximation ratio) on every truncated distribution F. I.e., $M^{\prime}(F) \geq M(F)$.

Proof. In a stochastic markup mechanism the price of the higher agent is a stochastic multiplicative factor $r \geq 1$ of the value of the lower agent (with ties broken randomly). To prove this theorem we must argue that (a) if the agents are not tied, then revenue improves if the lower agent loses, (b) if the agents are tied, then revenue is unaffected by random tie-breaking, and (c) any such scale-invariant mechanism looks to the higher-valued agent like a stochastic posted pricing with price that is a multiplicative factor (at least one) of the lower-valued agent's value.

To see (a), note that the revenue of the mechanism is equal to its virtual surplus (Theorem 2) and for triangle distributions only the highest value in the support of the distribution has positive virtual value. Thus, any mechanism that sells to a strictly-lower-valued agent can be improved by not selling to such an agent.

To see (b), note that for any i.i.d. distribution the revenue of any mechanism is invariant to randomly permuting the identities of the agents. Thus, we can assume random tie-breaking.

To see (c), recall that the family of incentive-compatible single-agent mechanisms is equivalent to the family of random price postings. Once we have ruled out selling to the lower-valued agent, the mechanism is a single-agent mechanism for the higher-valued agent (with price at least the lowervalued agent's value. By the assumption that the mechanism is scale invariant, the distribution of prices offered to the higher-valued agent must be multiplicative scalings of the lower-valued agent's value.

Next we will give a sequence of results that culminate in the observation that for any regular distribution and any stochastic markup mechanism with probability $\alpha$ at least $2 / 3$ on the secondprice auction (which includes the optimal mechanism from Theorem 44) either the triangulation of the distribution or the point mass $\operatorname{Tr}_{1}$ has (weakly) higher approximation ratio. As the notation indicates, the point mass distribution $\mathrm{Tri}_{1}$ is a triangle distribution.

Theorem 6. For i.i.d., two-agent, single-item environments and any regular distribution $F$ and any stochastic markup mechanism $M$ that places probability $\alpha \in[2 / 3,1]$ on the second-price auction, either the triangulation of the distribution $F^{\operatorname{Tri}}$ or the point mass $\operatorname{Tri}_{1}$ has (weakly) higher

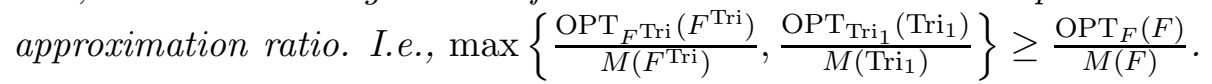

To prove this theorem we give a sequence of results showing that for any regular distribution, a corresponding truncated distribution is only worse; for any truncated distribution and a fixed 

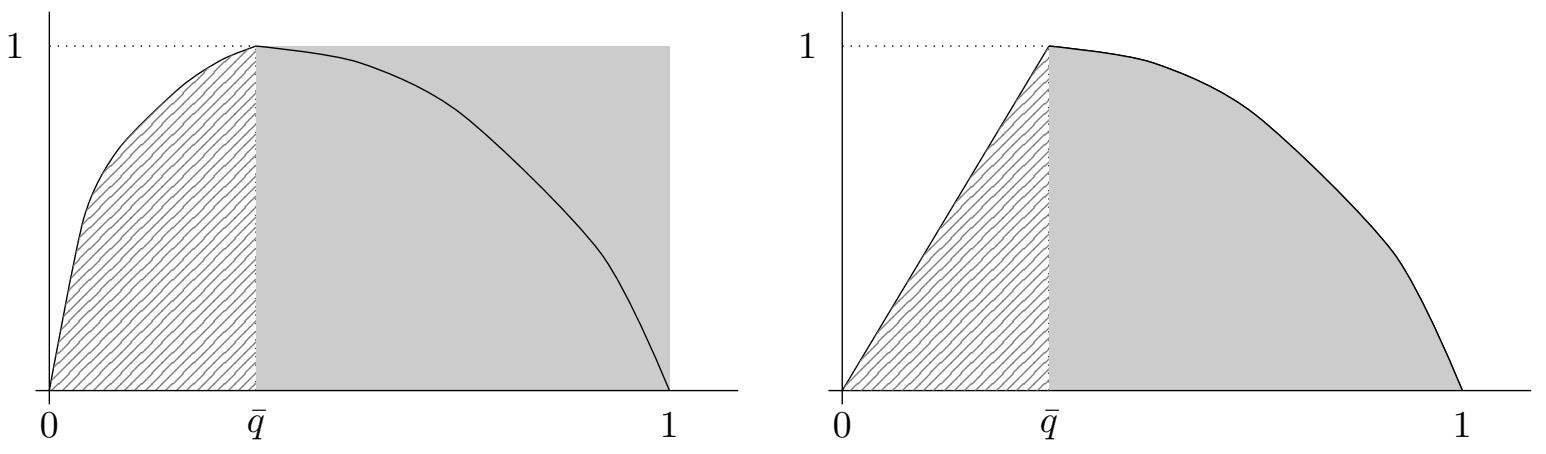

Figure 3: The illustration of the revenue decomposition of Lemma 7 for $M$ on distribution $F$ and truncation $F^{\prime}$ for the optimal mechanism and second-price auction. The thin black line on the left and right figures are the revenue curves corresponding to $F$ and $F^{\prime}$, respectively. The dashed area on the left represents $\mathrm{OPT}_{+}=\mathrm{SPA}_{+}$and the gray area on the left represents $\mathrm{OPT}_{-}=\mathrm{OPT}_{-}^{\prime}$. The dashed area on the right represents $\mathrm{OPT}_{+}^{\prime}=\mathrm{SPA}_{+}^{\prime}$ and the gray area on the right represents $\mathrm{SPA}_{-}^{\prime}=\mathrm{SPA}_{-}$.

stochastic markup mechanism (that mixes over $M_{1}$ and some $M_{r}$ ), a corresponding quadrilateral distribution (based on $r$ ) is only worse; and for any quadrilateral distribution, a corresponding triangle distribution (independent of $r$ ) is only worse. The theorem follows from combining these results. The first step assumes that the probability that the stochastic markup mechanism places on the second price auction is $\alpha \in[1 / 2,1]$; the last step further assumes that $\alpha \in[2 / 3,1]$.

To begin, the following lemma shows that the best response of the adversary to a relevant stochastic markup mechanism is a truncated distribution. Recall that by $\mathrm{Fu}$ et al. (2015) the prior-independent optimal mechanism is strictly better than a 2-approximation. On the other hand, any stochastic markup mechanism that places probability $\alpha$ on the second-price auction $M_{1}$ has prior-independent approximation at least $1 / \alpha$. Specifically, on the (degenerate) distribution that places all probability mass on 1, a.k.a. Tri $1_{1}$, the approximation factor of such a stochastic markup mechanism is exactly $1 / \alpha$. We conclude that all relevant stochastic markup mechanisms place probability $\alpha>1 / 2$ on the second-price auction. Thus, this lemma applies to all relevant mechanisms.

Lemma 7. For i.i.d., two-agent, single-item environments, any regular distribution $F$, and any stochastic markup mechanism $M$ that places probability $\alpha \in[1 / 2,1]$ on the second-price auction; either the truncation of the distribution $F^{\prime}$ or the point mass distribution $\mathrm{Tri}_{1}$ has (weakly) higher

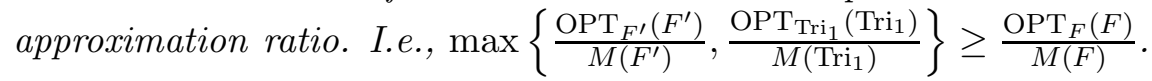

Proof. It can be assumed that the approximation of stochastic markup mechanism $M$ on distribution $F$ is at least $1 / \alpha$ (where $\alpha$ denotes the probability that $M$ places on the second-price auction). Notice that the revenue $M$ on the point mass on 1 (a truncated distribution) is $\alpha$ and the optimal revenue on this distribution is 1 . If the approximation factor $\operatorname{OPT}_{F}(F) / M(F)$ is less than $1 / \alpha$ then the point mass on 1 (a truncated distribution) achieves a higher approximation than $F$ and the lemma follows. For the remainder of the proof, assume that the approximation factor of mechanism $M$ on distribution $F$ is more than $1 / \alpha$. 
View the stochastic markup mechanism $M$ as a distribution over two mechanisms: the secondprice auction $M_{1}$ with probability $\alpha$, and $M_{*}$, a distribution over non-trivial markup mechanisms $M_{r}$ with $r>1$, with probability $1-\alpha$. The optimal mechanism is $\mathrm{OPT}_{F}$. Decompose the revenue from distribution $F$ across these three mechanisms as follows. Denote the monopoly quantile of $F$ by $\bar{q}$. See Figure 3 ,

- $\mathrm{OPT}_{+}$and $\mathrm{OPT}_{-}$give the expected revenue of the optimal mechanism from agents with values above and below the monopoly price (below and above the monopoly quantile $\bar{q}$ ).

- $\mathrm{SPA}_{+}=\mathrm{OPT}_{+}$and $\mathrm{SPA}_{-}$give the expected revenue of the second-price auction $M_{1}$ from agents with values above and below the monopoly price.

- $\mathrm{MKUP}_{+}$and MKUP - give the expected revenue of the stochastic markup mechanism $M_{*}$ from prices (strictly) above and (weakly) below the monopoly price.

Consider truncating the distribution $F$ at the monopoly quantile $\bar{q}$ to obtain $F^{\prime} \in \mathcal{F}^{\text {Trunc }}$. Define analogous quantities (with identities):

- $\mathrm{OPT}_{+}^{\prime}<\mathrm{OPT}_{+}$and $\mathrm{OPT}_{-}^{\prime}=\mathrm{OPT}_{-}$.

Identities follow from the geometric analysis of Lemma 3 .

- $\mathrm{SPA}_{+}^{\prime}=\mathrm{OPT}_{+}^{\prime}$ and $\mathrm{SPA}_{-}^{\prime}=\mathrm{SPA}_{-}$.

Identities follow from the geometric analysis of Lemma 3 ,

- $\mathrm{MKUP}_{+}^{\prime}=0$ and $\mathrm{MKUP}_{-}^{\prime}=\mathrm{MKUP}_{-}$.

Values above the monopoly price are not supported by the truncated distribution, so the revenue from those prices is zero. On the other hand, prices (weakly) below the monopoly price are bought with the exact same probability as the cumulative distribution function $F^{\prime}$ and $F$ are the same for these prices.

The remainder of the proof follows a straightforward calculation. Write the approximation ratio of $M$ on distribution $F$ (using the given identities) and rearrange:

$$
\begin{aligned}
\frac{\mathrm{OPT}_{F}(F)}{M(F)} & =\frac{\mathrm{OPT}_{+}+\mathrm{OPT}_{-}}{\alpha\left(\mathrm{OPT}_{+}+\mathrm{SPA}_{-}\right)+(1-\alpha)\left(\mathrm{MKUP}_{+}+\mathrm{MKUP}_{-}\right)} \\
& =\frac{\mathrm{OPT}_{+}+\left[\mathrm{OPT}_{-}\right]}{\alpha \mathrm{OPT}_{+}+\left[\alpha \mathrm{SPA}_{-}+(1-\alpha)\left(\mathrm{MKUP}_{+}+\mathrm{MKUP}_{-}\right)\right]}
\end{aligned}
$$

Since the approximation ratio on $F$ is at least $1 / \alpha$, the ratio of the first term in the numerator and denominator is at most the ratio of the remaining terms [in brackets]:

$$
\frac{1}{\alpha}=\frac{\mathrm{OPT}_{+}}{\alpha \mathrm{OPT}_{+}} \leq \frac{\left[\mathrm{OPT}_{-}\right]}{\left[\alpha \mathrm{SPA}_{-}+(1-\alpha)\left(\mathrm{MKUP}_{+}+\mathrm{MKUP}_{-}\right)\right]}
$$



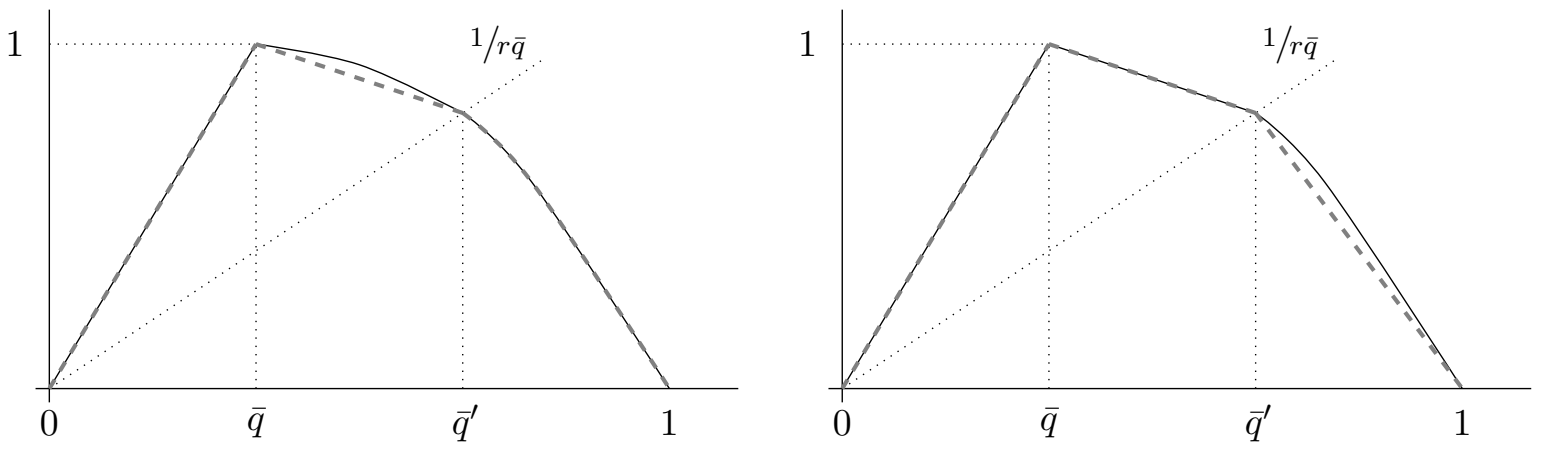

Figure 4: The main two steps of Lemma 9 are illustrated. In the first step (right-hand side), the revenue curves of distributions $F^{\text {Trunc }}$ (thin, solid, black) and $F^{\dagger}$ (thick, dashed, gray) are depicted. In the second step, the revenue curves of the distributions $F^{\dagger}$ (thin, solid, black) and $F^{\mathrm{Qr}}$ (thick, dashed, gray) are depicted. In both cases the revenue of the $r$-markup mechanism is is higher on the thin, solid, black curve than the thick, dashed, gray curve.

Now write the approximation ratio of $M$ on truncation $F^{\prime}$ (using the given identities) and bound:

$$
\begin{aligned}
\frac{\mathrm{OPT}_{F^{\prime}}\left(F^{\prime}\right)}{M\left(F^{\prime}\right)} & =\frac{\mathrm{OPT}_{+}^{\prime}+\left[\mathrm{OPT}_{-}\right]}{\alpha \mathrm{OPT}_{+}^{\prime}+\left[\alpha \mathrm{SPA}_{-}+(1-\alpha) \mathrm{MKUP}_{-}\right]} \\
& \geq \frac{\mathrm{OPT}_{+}^{\prime}+\left[\mathrm{OPT}_{-}\right]}{\alpha \mathrm{OPT}_{+}^{\prime}+\left[\alpha \mathrm{SPA}_{-}+(1-\alpha)\left(\mathrm{MKUP}_{+}+\mathrm{MKUP}_{-}\right)\right]} \\
& \geq \frac{\mathrm{OPT}_{+}+\left[\mathrm{OPT}_{-}\right]}{\alpha \mathrm{OPT}_{+}+\left[\alpha \mathrm{SPA}_{-}+(1-\alpha)\left(\mathrm{MKUP}_{+}+\mathrm{MKUP}_{-}\right)\right]} \\
& =\frac{\mathrm{OPT}_{F}(F)}{M(F)} .
\end{aligned}
$$

The calculation shows that, for any distribution $F$, the truncated distribution $F^{\prime}$ increases the approximation factor of the stochastic markup mechanism. Thus, the worst-case distribution is truncated.

The next step is to show that, among truncated distributions, the worst-case distribution for stochastic markup mechanisms are those with quadrilateral-shaped revenue curves, i.e., ones that are piecewise linear with three pieces (see Figure 1). Recall that for a truncated distribution at monopoly quantile $\bar{q}$, the upper bound of the support is a point mass on $1 / \bar{q}$.

Definition 12. A normalized quadrilateral distribution with parameters $\bar{q}, \bar{q}^{\prime}$ and $r$ with $r \geq 1$ and $\frac{\bar{q} r}{\bar{q} r+(1-\bar{q})} \leq \bar{q}^{\prime} \leq \min \{r \bar{q}, 1\}$, denoted by $\mathrm{Qr}_{\bar{q}, \bar{q}^{\prime}, r}$ is defined by quantile function as:

$$
Q_{\mathrm{Qr}_{\bar{q}, \bar{q}^{\prime}, r}}(v)= \begin{cases}\frac{\bar{q}^{\prime}}{\bar{q}^{\prime}+v r \overline{\bar{q}}\left(1-\bar{q}^{\prime}\right)} & v<1 / r \bar{q} \\ \frac{\bar{q}^{\prime}(\bar{q}(r-1)}{v r \bar{q}\left(\bar{q}^{\prime}-\bar{q}\right)+\left(r \bar{q}-\bar{q}^{\prime}\right)} & 1 / r \bar{q} \leq v \leq 1 / \bar{q} \\ 0 & 1 / \bar{q}<v\end{cases}
$$

The following lemma summarizes an analysis from Allouah and Besbes (2018) and is useful in bounding the revenue from markup mechanisms. 
Lemma 8 (Allouah and Besbes, 2018). Consider the r-markup mechanism, two i.i.d. regular agents with value distribution $F$, quantile $\bar{q}^{\prime}$ corresponding to the monopoly price divided by $r$, and the distribution $\tilde{F}$ that corresponds to $F$ ironed on $\left[\bar{q}^{\prime}, 1\right]$ : the virtual surplus from quantiles $\left[\bar{q}^{\prime}, 1\right]$ is higher for $F$ than for $\tilde{F}$.

Proof. The proof of this lemma is technical and non-trivial. It is given in the proof of Proposition 4 of Allouah and Besbes (2018).

The next lemma reduces the worst case distribution from the family of truncated distributions to the family of quadrilateral distributions. The reduction is illustrated in Figure 4, by showing that ironing the revenue curves sequentially within $\left[\bar{q}, \bar{q}^{\prime}\right]$ and $\left[\bar{q}^{\prime}, 1\right]$ decreases the revenue of the stochastic markup mechanism. The optimal revenue is not affected because it is obtained using a reserve price corresponding to the monopoly quantile $\bar{q}$ and it is agnostic to the shape of the revenue curve for $q>\bar{q}$.

Lemma 9. For i.i.d., two-agent, single-item environments, any truncated distribution $F^{\text {Trunc, }}$ and any stochastic markup mechanism $M_{\alpha, r}$ with probability $\alpha$ on the second-price auction $M_{1}$ and probability $1-\alpha$ on non-trivial markup mechanism $M_{r}$; there is a quadrilateral distribution $F^{\mathrm{Qr}}$ with the same optimal revenue and (weakly) lower revenue in $M_{\alpha, r}$. I.e., $\mathrm{OPT}_{F} \mathrm{Qr}\left(F^{\mathrm{Qr}}\right)=$ $\mathrm{OPT}_{F^{\text {Trunc }}}\left(F^{\text {Trunc }}\right)$ and $M_{\alpha, r}\left(F^{\mathrm{Qr}}\right) \leq M_{\alpha, r}\left(F^{\text {Trunc }}\right)$.

Proof. On any normalized truncated distribution with monopoly quantile $\bar{q}$, the optimal revenue is $2-\bar{q}$ (Lemma 44). Thus, to prove the lemma it is sufficient to show that for any truncated distribution $F^{\text {Trunc }} \in \mathcal{F}^{\text {Trunc }}$ with monopoly quantile $\bar{q}$ there is a normalized quadrilateral distribution $F^{\mathrm{Qr}} \in$ $\mathcal{F}^{\mathrm{Qr}} \subset \mathcal{F}^{\text {Trunc }}$ with monopoly quantile $\bar{q}$ and lower revenue in $M_{\alpha, r}$.

The quadrilateral distribution $F^{\mathrm{Qr}}$ is obtained by ironing $F^{\text {Trunc }}$ on $[\bar{q}, \bar{q}]$ and $\left[\bar{q}^{\prime}, 1\right]$ where quantile $\bar{q}^{\prime}$ satisfies $V_{F^{\text {Trunc }}}(\bar{q})=r V_{F^{\text {Trunc }}}\left(\bar{q}^{\prime}\right)$. We consider an intermediary distribution $F^{\dagger}$ that is $F^{\text {Trunc }}$ ironed only on $[\bar{q}, \bar{q}]$. See Figure 4, The proof approach is to show that $M_{\alpha, r}\left(F^{\text {Trunc }}\right)>$ $M_{\alpha, r}\left(F^{\dagger}\right)>M_{\alpha, r}\left(F^{\mathrm{Qr}}\right)$.

As $M_{\alpha, r}$ is a convex combination of the second-price auction $M_{1}$ and the $r$-markup mechanism $M_{r}$. It suffices to show the inequalities above hold for both auctions. In fact, the result holds for the second-price auction from the geometric analysis of revenue of Lemma 3 . The revenue of the secondprice auction for two i.i.d. agents is twice the area under the revenue curve. As the revenue curve has strictly smaller area from $F^{\text {Trunc }}$ to $F^{\dagger}$ to $F^{\mathrm{Qr}}$, we have $M_{1}\left(F^{\text {Trunc }}\right)>M_{1}\left(F^{\dagger}\right)>M_{1}\left(F^{\mathrm{Qr}}\right)$. Below, we analyze the $r$-markup mechanism $M_{r}$.

The following price-based analysis shows that $M_{r}\left(F^{\text {Trunc }}\right)>M_{r}\left(F^{\dagger}\right)$ :

- The revenue from quantiles in $[0, \bar{q}]$ is unchanged.

These quantiles are offered prices from quantiles in $\left[\bar{q}^{\prime}, 1\right]$. The values of quantiles $[0, \bar{q}]$ and $\left[\bar{q}^{\prime}, 1\right]$ are the same for both distributions; thus, the revenue is unchanged.

- The revenue from quantiles in $[\bar{q}, \bar{q}]$ decreases.

These quantiles are offered prices from quantiles in $\left[\bar{q}^{\prime}, 1\right]$. For the distribution $F^{\dagger}$ relative to $F^{\text {Trunc: }}$ Values are lower at any quantile $q \in[\bar{q}, \bar{q}]$; the distribution of prices (from quantiles in $\left.\left[\bar{q}^{\prime}, 1\right]\right)$ is the same. Thus, revenue is lower.

- The revenue from quantiles in $\left[\bar{q}^{\prime}, 1\right]$ is unchanged. 


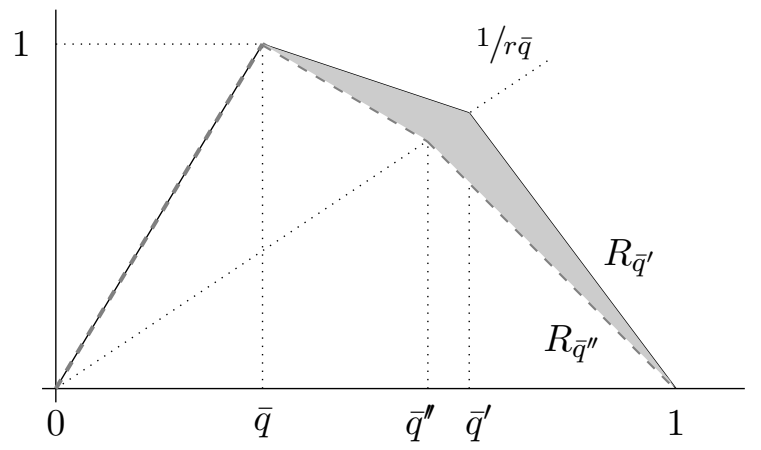

Figure 5: Illustrating the proof of Lemma 10, the difference of revenue for second price auction $M_{1}$ on revenue curves $R_{\bar{q}^{\prime}}$ and $R_{\bar{q}^{\prime \prime}}$, which respectively correspond to quadrilateral distributions $\mathrm{Qr}_{\bar{q}, \bar{q}^{\prime}, r}$ and $\mathrm{Qr}_{\bar{q}, \bar{q}^{\prime \prime}, r}$, is equal to twice of the gray area, which is at least $\bar{q}^{\prime \prime}-\bar{q}^{\prime}$. Moreover, the difference of revenue for the $r$-markup mechanism $M_{r}$ on revenue curves $R_{\bar{q}^{\prime}}$ and $R_{\bar{q}^{\prime \prime}}$ is at most $2\left(\bar{q}^{\prime \prime}-\bar{q}^{\prime}\right.$.

These quantiles are in $\left[\bar{q}^{\prime}, 1\right]$ and are offered prices from quantiles in $\left[\bar{q}^{\prime}, 1\right]$. The distributions are the same for these quantiles; thus, the revenue is unchanged.

The following virtual-surplus-based analysis shows that $M_{r}\left(F^{\dagger}\right)>M_{r}\left(F^{\mathrm{Qr}}\right)$ :

- The virtual surplus of quantiles in $[0, \bar{q}]$ is unchanged.

These quantiles have the same virtual values under the two distributions and the same probability of winning, i.e., $1-\bar{q}^{\prime}$ (when the other agent's quantile is in $\left[\bar{q}^{\prime}, 1\right]$.

- The virtual surplus of quantiles in $[\bar{q}, \bar{q}]$ is decreased.

Their prices come from quantiles in $\left[\bar{q}^{\prime}, 1\right]$ which are decreased; thus, their probabilities of winning are increased. Their virtual values are negative, so these increased probabilities of winning result in decreased virtual surplus.

- The virtual surplus of quantiles in $\left[\bar{q}^{\prime}, 1\right]$ is decreased.

This result is given by Lemma 8 ,

We complete the proof of Theorem 6 by showing that triangle distributions lead to lower revenue than quadrilateral distributions. The intuition of the proof is illustrated in Figure 5. For any $r>1$ and any stochastic markup mechanism $M_{\alpha, r}$ with probability $\alpha \in[2 / 3,1]$, consider a family of quadrilateral distributions $\mathrm{Qr}_{\bar{q}, \bar{q}^{\prime}, r}$ parameterized by $\bar{q}^{\prime}$. The optimal revenue is again not affected by $\bar{q}^{\prime}$ while the revenue of $M_{\alpha, r}$ is monotone increasing in $\bar{q}^{\prime}$. Thus the approximation ratio of $M_{\alpha, r}$ is maximized by minimal $\bar{q}^{\prime}$ for which the degenerate quadrilateral $\mathrm{Qr}_{\bar{q}, \bar{q}^{\prime}, r}$ is a triangle.

Lemma 10. For i.i.d., two-agent, single-item environments, normalized quadrilateral distribution $\mathrm{Qr}_{\bar{q}, \bar{q}^{\prime}, r}$, and stochastic markup mechanism $M_{\alpha, r}$ with probability $\alpha \in[2 / 3,1]$ on the second-price auction $M_{1}$ and probability $1-\alpha$ on non-trivial markup mechanism $M_{r}$; the triangle distribution $\operatorname{Tri}_{\bar{q}}$ has the same optimal revenue and (weakly) lower revenue in $M_{\alpha, r}$. I.e., $\mathrm{OPT}_{\operatorname{Tri}}\left(\operatorname{Tri}_{\bar{q}}\right)=$ $\mathrm{OPT}_{\mathrm{Qr}_{\bar{q}, \bar{q}^{\prime}, r}}\left(\mathrm{Qr}_{\bar{q}, \bar{q}^{\prime}, r}\right)$ and $M_{\alpha, r}\left(\operatorname{Tri}_{\bar{q}}\right) \leq M_{\alpha, r}\left(\mathrm{Qr}_{\bar{q}, \bar{q}^{\prime}, r}\right)$.

Proof. By Lemma 4, the optimal revenues for quadrilateral distribution $\mathrm{Qr}_{\bar{q}, \bar{q}^{\prime}, r}$ and triangle distribution $\operatorname{Tri}_{\bar{q}}$ are the same (and equal to $2-\bar{q}$ ). To show that the revenue of $M_{\alpha, r}$ is worse on 
$\operatorname{Tri}_{\bar{q}}$ than $\mathrm{Qr}_{\bar{q}, \bar{q}^{\prime}, r}$, it suffices to show that the revenue on $\mathrm{Qr}_{\bar{q}, \bar{q}^{\prime}, r}$ is monotonically increasing in $\bar{q}^{\prime}$. Specifically the minimum revenue is when the quadrilateral distribution is degenerately equal to the triangle distribution.

The proof strategy is to lower bound the partial derivative with respect to $\bar{q}^{\prime}$ of the revenues of $r$-markup mechanism and the second-price auction for quadrilateral distributions $\mathrm{Qr}_{\bar{q}, \bar{q}^{\prime}, r}$ as

$$
\begin{aligned}
& \frac{\partial M_{r}\left(\mathrm{Qr}_{\bar{q}, \bar{q}^{\prime}, r}\right)}{\partial \bar{q}^{\prime}} \geq-2, \\
& \frac{\partial M_{1}\left(\mathrm{Qr}_{\bar{q}, \bar{q}^{\prime}, r}\right)}{\partial \bar{q}^{\prime}} \geq 1 .
\end{aligned}
$$

Thus, for mechanism $M_{\alpha, r}$ with $\alpha \geq 2 / 3$, we have

$$
\frac{\partial M_{\alpha, r}\left(\mathrm{Qr}_{\bar{q}, \bar{q}^{\prime}, r}\right)}{\partial \bar{q}^{\prime}} \geq \alpha-2(1-\alpha) \geq 0
$$

and revenue is minimized with the smallest choice of $\bar{q}^{\prime}$ for which quadrilateral distribution $\mathrm{Qr}_{\bar{q}, \bar{q}^{\prime}, r}$ is degenerately a triangle distribution. It remains to prove bounds (3) and (4).

For simplicity, since in this section the only parameter we change in distribution $\mathrm{Qr}_{\bar{q}, \bar{q}^{\prime}, r}$ is $\bar{q}^{\prime}$, we introduce the notation $P_{\bar{q}^{\prime}}(v)$ to denote the revenue of posting price $v$, and $V_{\bar{q}^{\prime}}(q)$ to denote the price $v$ given quantile $q$ when the distribution is $\mathrm{Qr}_{\bar{q}, \bar{q}^{\prime}, r}$. The proof is illustrated in Figure 5.

We now prove bound (3). For any pair of quadrilateral distributions $\mathrm{Qr}_{\bar{q}, \bar{q}^{\prime}, r}$ and $\mathrm{Qr}_{\bar{q}, \bar{q}^{\prime \prime}, r}$ with $\bar{q}^{\prime \prime} \geq \bar{q}^{\prime}$, we analyze the difference in revenue for posting price $r \cdot v_{(2)}$.

$$
\begin{aligned}
& M_{r}\left(\mathrm{Qr}_{\bar{q}, \bar{q}^{\prime \prime}, r}\right)-M_{r}\left(\mathrm{Qr}_{\bar{q}, \bar{q}^{\prime}, r}\right) \\
& \quad=2 \int_{\bar{q}^{\prime \prime}}^{1} P_{\bar{q}^{\prime \prime}}\left(r \cdot V_{\bar{q}^{\prime \prime}}(q)\right) d q-2 \int_{\bar{q}^{\prime}}^{1} P_{\bar{q}^{\prime}}\left(r \cdot V_{\bar{q}^{\prime}}(q)\right) d q \\
& \quad \geq 2 \int_{\bar{q}^{\prime \prime}}^{1} P_{\bar{q}^{\prime \prime}}\left(r \cdot V_{\bar{q}^{\prime \prime}}(q)\right) d q-2 \int_{\bar{q}^{\prime \prime}}^{1} P_{\bar{q}^{\prime}}\left(r \cdot V_{\bar{q}^{\prime}}(q)\right) d q-2\left(\bar{q}^{\prime \prime}-\bar{q}^{\prime}\right) \\
& \quad \geq 2 \int_{\bar{q}^{\prime \prime}}^{1} P_{\bar{q}^{\prime}}\left(r \cdot V_{\bar{q}^{\prime \prime}}(q)\right) d q-2 \int_{\bar{q}^{\prime \prime}}^{1} P_{\bar{q}^{\prime}}\left(r \cdot V_{\bar{q}^{\prime}}(q)\right) d q-2\left(\bar{q}^{\prime \prime}-\bar{q}^{\gamma}\right) \\
& \quad \geq-2\left(\bar{q}^{\prime \prime}-\bar{q}^{\prime}\right) .
\end{aligned}
$$

The first equality is constructed as follows: Both agents face a random price that is $r$ times the value of the other agent who has quantile $q$ drawn from $U[0,1]$. The revenue from this price is given by, e.g., $P_{\bar{q}^{\prime}}\left(r \cdot V_{\bar{q}^{\prime}}(q)\right)$ which is 0 when $q \leq \bar{q}^{\prime}$. The first inequality holds because $P_{\bar{q}^{\prime}}\left(r \cdot V_{\bar{q}^{\prime}}(q)\right) \leq 1$ for any quantile $q$. The second inequality holds since the revenue from revenue curve $P_{\bar{q}^{\prime \prime}}$ is weakly higher than from revenue curve $P_{\bar{q}^{\prime}}$ for any value $v$. The third inequality holds because (a) the prices of the first integral are higher than the prices of the second integral, i.e., $V_{\bar{q}^{\prime \prime}}(q) \geq V_{\bar{q}^{\prime}}(q)$ for every $q$, and (b) because these prices are below the monopoly price for distribution $\mathrm{Qr}_{\bar{q}, \bar{q}^{\prime \prime}, r}$ and so higher prices give higher revenue.

Therefore, we have

$$
\frac{\partial M_{r}\left(\mathrm{Qr}_{\bar{q}, \bar{q}^{\prime}, r}\right)}{\partial \bar{q}^{\prime}}=\lim _{\bar{q}^{\prime \prime} \rightarrow \bar{q}^{\prime}} \frac{M_{r}\left(\mathrm{Qr}_{\bar{q}, \bar{q}^{\prime \prime}, r}\right)-M_{r}\left(\mathrm{Qr}_{\bar{q}, \bar{q}^{\prime}, r}\right)}{\bar{q}^{\prime \prime}-\bar{q}^{\prime}} \geq-2 .
$$


We now prove bound (4). The revenue of the second price auction for two i.i.d. agents is twice the area under the revenue curve (Lemma 3). For quadrilateral distribution $\mathrm{Qr}_{\bar{q}, \bar{q}^{\prime}, r}$ this revenue is calculated as:

$$
\begin{aligned}
M_{1}\left(\mathrm{Qr}_{\bar{q}, \bar{q}^{\prime}, r}\right) & =2 \int_{0}^{1} R_{\bar{q}^{\prime}}(q) d q \\
& =2 \int_{0}^{\bar{q}} R_{\bar{q}^{\prime}}(q) d q+2 \int_{\bar{q}}^{\bar{q}^{\prime}} R_{\bar{q}^{\prime}}(q) d q+2 \int_{\bar{q}^{\prime}}^{1} R_{\bar{q}^{\prime}}(q) d q \\
& =\bar{q}+\left(\bar{q}^{\prime}-\bar{q}\right)\left(1+\frac{\bar{q}^{\prime}}{r \cdot \bar{q}}\right)+\left(1-\bar{q}^{\prime}\right) \frac{\bar{q}^{\prime}}{r \cdot \bar{q}} \\
& =\bar{q}^{\prime}+(1-\bar{q}) \frac{\bar{q}^{\prime}}{r \cdot \bar{q}} .
\end{aligned}
$$

Therefore, we have

$$
\frac{\partial M_{1}\left(\mathrm{Qr}_{\bar{q}, \bar{q}^{\prime}, r}\right)}{\partial \bar{q}^{\prime}}=1+\frac{1-\bar{q}}{r \cdot \bar{q}} \geq 1
$$

\section{Sub-optimality of Relaxed Benchmark Design}

In this section, we will show that the relaxed benchmark design program $(\bar{\gamma})$ is not generally equal to the benchmark design program $(\sqrt{\gamma})$ by considering the revenue maximization problem for two agents with i.i.d. regular distributions. Since benchmark optimization and prior-independent mechanism design are equivalent problems (see Section 3. Theorem 1), a gap between the objective values $\bar{\gamma}$ and $\gamma$ of programs $(\bar{\gamma})$ and $(\bar{\gamma})$ is implied by exhibiting a benchmark with lower objective value in program $(\bar{\gamma})$ than the approximation achieved by the optimal prior-independent mechanism, i.e., the solution to program $(\beta)$.

We first establish a gap in the value of these programs under the restriction to triangle distributions.

Theorem 7. For i.i.d. triangle distributions, two-agent, single-item environments and scale-invariant, incentive-compatible mechanisms the heuristic benchmark optimization program ( $\bar{\gamma}$ ) has a strictly smaller objective value than the benchmark optimization program $(\sqrt{\gamma})$, i.e., $\bar{\gamma}<\gamma$.

This section follows the notations and definitions introduced in Section 3 , Let $M_{\alpha^{*}, r^{*}}$ be the approximately optimal prior-independent mechanism with numerical parameters $\alpha^{*}=0.80564048, r^{*}=$ 2.4469452 (Theorem 3). We first define the pseudo-mechanism $\tilde{M}$ as the affine combination of mechanisms with revenue $\tilde{M}(\boldsymbol{v})=(1+\delta) M_{\alpha^{*}, r^{*}}(\boldsymbol{v})-\delta M_{1.1}(\boldsymbol{v})$, where $\delta>0$ is a small constant. Recall that $\mathcal{F}^{\text {Tri }}$ is the family of triangle distributions. We show that

1. $\forall F \in \mathcal{F}^{\operatorname{Tri}}, \tilde{M}(F) \leq \operatorname{OPT}_{F}(F)$;

2. $\forall F \in \mathcal{F}^{\text {Tri }}, \beta^{\prime} \tilde{M}(F) \geq \operatorname{OPT}_{F}(F)$, where $\beta^{\prime}<\beta$.

Letting $B(\boldsymbol{v})=\beta^{\prime} \tilde{M}(\boldsymbol{v})$, benchmark $B$ is normalized for all triangle distributions and the approximation of optimal payoff against benchmark $B$ is $\beta^{\prime}<\beta=\gamma$. Therefore, the approximation of the optimal heuristic benchmark is $\bar{\gamma} \leq \beta^{\prime}<\gamma$ and Theorem 7 holds.

We first prove that the first statement holds by showing a more general lemma for regular distributions with proof deferred at the end of the section. 
Lemma 11. For i.i.d., two-agent, regular, single-item environments, any constants $1 \leq r_{1} \leq r_{2} \leq$ $r_{3}$, and any constants $a, b, c \geq 0$ such that $a+b-c \leq 1, a \geq c, b \geq c$ :

$$
\tilde{M}(F)=a M_{r_{1}}(F)+b M_{r_{3}}(F)-c M_{r_{2}}(F) \leq \mathrm{OPT}_{F}(F) .
$$

Our approach to show that the second statement holds is as follows. Recall that the benchmark program $(\gamma)$ has value 1.9068943. It will suffice then to show that on triangle distributions defined by monopoly quantile $\bar{q}$ close to $\bar{q}^{*}=0.0931057$, where the prior-independent optimal mechanism $M_{\alpha^{*}, r^{*}}$ obtains revenue at least 1.9068943 , that $M_{1.1}$ performs very badly (specifically, it is worse than a 2-approximation). Moreover, on distributions with monopoly quantile $\bar{q}$ far from $\bar{q}^{*}$, mechanism $M_{\alpha^{*}, r^{*}}$ does much better than it does on worst case distributions (specifically, it is better than a 1.9041 approximation). The theorem will follow by combining these observations.

The following lemmas establish the two main steps of the argument. These lemmas are based on our formula for the revenue $M_{r}\left(\operatorname{Tri}_{\bar{q}}\right)$ as given by Lemma 6. We evaluate the formula for monopoly quantiles $\bar{q}$ in a grid and then use the smoothness of the revenue formula that is established in Appendix $\mathrm{A}$ to show that the revenue for all monopoly quantiles in the desired range satisfy the desired bound. The formal proof of these lemmas is given in Appendix B.

Lemma 12. For i.i.d., two-agent, single-item environments, and any quantile $\bar{q} \in[0.05,0.25]$, the approximation of mechanism $M_{1.1}$ on triangle distribution $\operatorname{Tr}_{\bar{q}}$ is at least 2.

Lemma 13. For i.i.d., two-agent, single-item environments, and any quantile $\bar{q} \notin[0.05,0.25]$, the approximation of mechanism $M_{\alpha^{*}, r^{*}}$ on triangle distribution $\operatorname{Tr}_{\bar{q}}$ is at most 1.9041.

To complete the proof of Theorem 7 , we combine these lemmas as follows. Setting $\delta=0.00154$, for $\bar{q} \in[0.05,0.25]$, by Lemma 12 and Theorem 3, we have

$$
\begin{aligned}
\frac{\mathrm{OPT}_{\operatorname{Tri}_{\bar{q}}}\left(\operatorname{Tri}_{\bar{q}}\right)}{\mathbf{E}_{\boldsymbol{v} \sim F}[\tilde{M}(\boldsymbol{v})]} & =\frac{\mathrm{OPT}_{\operatorname{Tri}_{\bar{q}}}\left(\operatorname{Tri}_{\bar{q}}\right)}{(1+\delta) M_{\alpha^{*}, r^{*}}\left(\operatorname{Tri}_{\bar{q}}\right)-\delta M_{1.1}\left(\operatorname{Tri}_{\bar{q}}\right)} \\
& \leq \frac{\mathrm{OPT}_{\operatorname{Tri}_{\bar{q}}}\left(\operatorname{Tri}_{\bar{q}}\right)}{\frac{1+\delta}{1.9068943} \mathrm{OPT}_{\operatorname{Tri}_{\bar{q}}}\left(\operatorname{Tri}_{\bar{q}}\right)-\frac{\delta}{2} \mathrm{OPT}_{\operatorname{Tri}_{\bar{q}}}\left(\operatorname{Tr}_{\bar{q}}\right)} \leq 1.90676 .
\end{aligned}
$$

For $\bar{q} \notin[0.05,0.25]$, we have $M_{1.1}\left(\operatorname{Tri}_{\bar{q}}\right) \leq \mathrm{OPT}_{\operatorname{Tr}} \mathrm{i}_{\bar{q}}\left(\operatorname{Tr}_{\bar{q}}\right)$, and by Lemma 13 ,

$$
\begin{aligned}
\frac{\operatorname{OPT}_{\operatorname{Tr} \mathrm{i}_{\bar{q}}}\left(\operatorname{Tri}_{\bar{q}}\right)}{\mathbf{E}_{\boldsymbol{v} \sim F}[\tilde{M}(\boldsymbol{v})]} & =\frac{\operatorname{OPT}_{\operatorname{Tr} \mathrm{i}_{\bar{q}}}\left(\operatorname{Tri}_{\bar{q}}\right)}{(1+\delta) M_{\alpha^{*}, r^{*}}\left(\operatorname{Tri}_{\bar{q}}\right)-\delta M_{1.1}\left(\operatorname{Tri}_{\bar{q}}\right)} \\
& \leq \frac{\mathrm{OPT}_{\operatorname{Tr}}\left(\operatorname{Tri}_{\bar{q}}\right)}{\frac{1+\delta}{1.9041} \mathrm{OPT}_{\operatorname{Tr} i_{\bar{q}}}\left(\operatorname{Tri}_{\bar{q}}\right)-\delta \mathrm{OPT}_{\operatorname{Tri}_{\bar{q}}}\left(\operatorname{Tr}_{\bar{q}}\right)} \leq 1.90676 .
\end{aligned}
$$

Therefore, the gap between program $(\bar{\gamma})$ and $(\bar{\gamma})$ is at least $10^{-4}$ for triangle distributions.

Theorem 7 can be generalized, from the restriction to triangle distributions, to regular distributions. This result is summarized below in Theorem 8 and its proof is given in Appendix B. The argument generalizes Lemma 12 and Lemma 13 to give bounds on markup mechanisms' revenues for regular distributions that are close to or far from the worst-case triangle distribution $\operatorname{Tri}_{\bar{q}^{*}}$. A key part of the proof is an appropriate definition of closeness. 


\begin{tabular}{|c|c|c|c|}
\hline & {$[0, \bar{q}]$} & {$\left[\bar{q}, \bar{q}^{\prime}\right]$} & {$\left[\bar{q}^{\prime}, 1\right]$} \\
\hline $\bar{R}_{F}\left(V_{F}(q) \cdot r_{1}\right)$ & $\bar{R}_{F}\left(V_{F}(q)\right)$ & 1 & 1 \\
\hline$-\bar{R}_{F}\left(V_{F}(q) \cdot r_{2}\right)$ & $-\bar{R}_{F}\left(V_{F}(q) \cdot r_{3}\right)$ & $-\bar{R}_{F}\left(V_{F}(q) \cdot r_{3}\right)$ & $-\bar{R}_{F}\left(V_{F}(q) \cdot r_{1}\right)$ \\
\hline $\bar{R}_{F}\left(V_{F}(q) \cdot r_{3}\right)$ & $\bar{R}_{F}\left(V_{F}(q)\right)$ & 1 & 1 \\
\hline
\end{tabular}

Figure 6: Bounds on revenues for the three markup mechanisms in three quantile ranges are shown. As $a$ and $b$ are both at least $c$, we can combine bounds $M_{r_{2}}$ with appropriate bounds for $M_{r_{1}}$ and $M_{r_{3}}$ to obtain: $b \bar{R}_{F}\left(V_{F}(q) \cdot r_{3}\right)-c \bar{R}_{F}\left(V_{F}(q) \cdot r_{2}\right) \leq(b-c) \bar{R}_{F}\left(V_{F}(q)\right)$ for $q \in[0, \bar{q}]$ and $a \bar{R}_{F}\left(V_{F}(q) \cdot r_{1}\right)+b \bar{R}_{F}\left(V_{F}(q) \cdot r_{3}\right)-c \bar{R}_{F}\left(V_{F}(q) \cdot r_{2}\right) \leq(a+b-c)$ for $q \in[\bar{q}, 1]$.

Theorem 8. For i.i.d., regular, two-agent, single-item environments and scale-invariant, incentivecompatible mechanisms the heuristic benchmark optimization program $(\bar{\gamma})$ has a strictly smaller objective value than the benchmark optimization program $(\bar{\gamma})$, i.e., $\bar{\gamma}<\gamma$.

Note that the parameters for the benchmark and the analysis of Theorem 7 and Theorem 8 are not optimized for the relaxed benchmark program. The main message of this section is just to show that the gap exists and that the relaxed benchmark program is inappropriate for optimizing benchmarks.

Proof of Lemma 11. Let $\bar{q}$ be the monopoly quantile of normalized distribution $F$ (i.e., with monopoly revenue 1). For convenience, denote the revenue as a function of price as $\bar{R}_{F}(v)=R_{F}\left(Q_{F}(v)\right)$. Let $\bar{q}^{\prime}=Q_{F}\left(V_{F}(\bar{q}) / r_{2}\right)$ be the quantile that corresponds to the monopoly reserve divided by $r_{2}$. The proof follows from the bounds in Figure 6 (derived below) and the following analysis. For $q \in[0, \bar{q}]$, combine the bounds in Figure 6 for $M_{r_{2}}$ and $M_{r_{3}}$ to obtain:

$$
b \bar{R}_{F}\left(V_{F}(q) \cdot r_{3}\right)-c \bar{R}_{F}\left(V_{F}(q) \cdot r_{2}\right) \leq(b-c) \bar{R}_{F}\left(V_{F}(q)\right) .
$$

Add the bound for $M_{r_{1}}$ :

$$
a \bar{R}_{F}\left(V_{F}(q) \cdot r_{1}\right)+b \bar{R}_{F}\left(V_{F}(q) \cdot r_{3}\right)-c \bar{R}_{F}\left(V_{F}(q) \cdot r_{2}\right) \leq(a+b-c) \bar{R}_{F}\left(V_{F}(q)\right) .
$$

Similarly, for $q \in[\bar{q}, 1]$ combine bounds in Figure 6 for $M_{r_{2}}$ with those for $M_{r_{1}}$ or $M_{r_{3}}$ (depending on $q$ ) to obtain:

$$
a \bar{R}_{F}\left(V_{F}(q) \cdot r_{1}\right)+b \bar{R}_{F}\left(V_{F}(q) \cdot r_{3}\right)-c \bar{R}_{F}\left(V_{F}(q) \cdot r_{2}\right) \leq(a+b-c) .
$$

Thus, we see the revenue of the pseudo mechanism is,

$$
\begin{aligned}
\tilde{M}(F) & =2 \int_{0}^{1}\left[a \bar{R}_{F}\left(V_{F}(q) \cdot r_{1}\right)+b \bar{R}_{F}\left(V_{F}(q) \cdot r_{3}\right)-c \bar{R}_{F}\left(V_{F}(q) \cdot r_{2}\right)\right] d q \\
& \leq 2(a+b-c)\left[\int_{0}^{\bar{q}} \bar{R}_{F}\left(V_{F}(q)\right) d q+(1-\bar{q})\right] \\
& =\operatorname{OPT}_{F}(F) .
\end{aligned}
$$

The proof then follows from the inequalities of Figure [6 which we now derive. The distribution $F$ is regular and by definition its revenue curve $R_{F}(q)$ is concave in $q$ (Figure 7). Therefore, $R_{F}(q)$ is increasing in $q$ for any quantile smaller than the monopoly quantile $\bar{q}$, i.e., $q \leq \bar{q}$, and $R_{F}(q)$ is decreasing in $q$ for any quantile larger than the monopoly quantile $\bar{q}$, i.e., $q \geq \bar{q}$. Since the quantile 


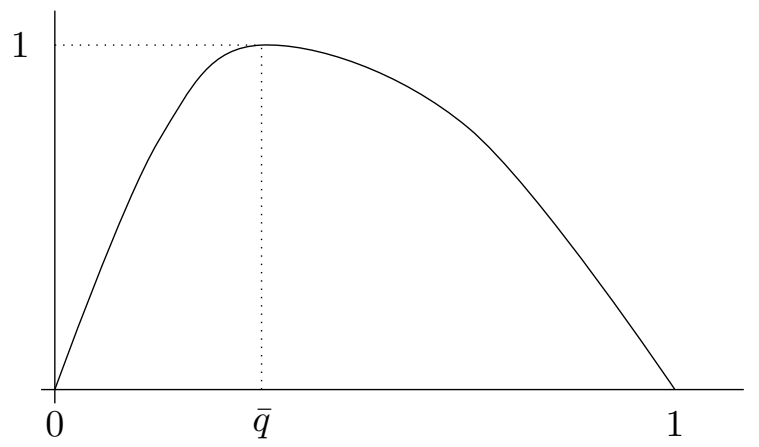

Figure 7: For regular distributions, $R_{F}(q)$ is increasing in $q$ for any quantile $q \leq \bar{q}$, and $R_{F}(q)$ is decreasing in $q$ for any quantile $q \geq \bar{q}$.

is decreasing in values, we have that $\bar{R}_{F}(v)$ is decreasing in $v$ for any value $V_{F}(\bar{q})$, i.e., $v \geq V_{F}(\bar{q})$, and $\bar{R}_{F}(v)$ is increasing in $v$ for any value $V_{F}(\bar{q})$, i.e., $v \leq V_{F}(\bar{q})$. The $[0, \bar{q}]$ column of Figure 6 follows from this monotonicity of $\bar{R}_{F}(\cdot)$. The $M_{r_{2}}$ row also follows from this monotonicity with the observation that $\bar{q}^{\prime}$ is defined so $V_{F}\left(\bar{q}^{\prime}\right) \cdot r_{2}$ equals the monopoly price $V_{F}(\bar{q})$. Thus, for $q<\bar{q}^{\prime}$, higher prices such as $V_{F}(q) \cdot r_{3}$ give lower revenue than $V_{F}(q) \cdot r_{2}$; and for $q \geq \bar{q}^{\prime}$ lower prices such as $V_{F}(q) \cdot r_{1}$ give lower revenue than $V_{F}(q) \cdot r_{2}$ (thus, the negated revenues are upper bounds). The remaining entries of 1 in Figure 6 follow from normalization which gives $\bar{R}_{F}(v) \leq 1$ for all prices $v$.

\section{Prior-free versus Prior-independent Expert Learning}

A main result of the paper, given in Section 2, is that optimal benchmark design, as we have defined it, is equivalent to prior-independent optimization. Moreover, the optimal prior-free algorithm for the optimal benchmark is the optimal prior-independent algorithm. A consequence of these results is that there is no added robustness from the prior-free framework over the prior-independent framework. In this section we observe, by an example of expert learning, that this potential lack of robustness is serious and the optimal prior-independent algorithm can perform much worse than the standard algorithms that are known to approximate the standard prior-free benchmark. These observations are straightforward from the perspective of the expert learning literature; we discuss them in detail so as to map them onto the framework of Section 2 and give formal proofs for completeness. (Moreover, Appendix D parallels Section 4 shows that the relaxed benchmark design program is not without loss of generality for expert learning.)

We consider the binary-reward variant of the canonical online expert learning problem. A single player plays a repeated game against Nature for $n$ rounds. In each round $t$, each expert $j$ from a discrete set $\{1, \ldots, k\}$ will receive a binary reward $v_{t, j} \in\{0,1\}$. Thus, the input space is $\mathcal{V}=\left[\{0,1\}^{k}\right]^{n}$. Before rewards are realized, the player chooses to "follow" a (possibly randomized) expert for the round, and receives a reward (possibly in expectation) equal to the reward of the followed expert. When the round concludes, the player gets to observe the rewards of all experts, including those not followed by the player. The player's algorithm is $M$ which outputs distributions $M_{t}(\boldsymbol{v})$ over experts using only the history $\left(v_{1}, \ldots, v_{t-1}\right)$ in each round $t$. The class of all such online algorithms is denoted by $\mathcal{M}^{\mathrm{OL}}$ and the performance of an online algorithm $M \in \mathcal{M}^{\mathrm{OL}}$ on input $\boldsymbol{v}$ 
is:

$$
M(\boldsymbol{v})=\sum_{t=1}^{n} \mathbf{E}_{j \sim M_{t}(\boldsymbol{v})}\left[v_{t, j}\right]
$$

As described in Section 2 we can define Bayesian, prior-independent, and prior-free versions of the expert learning problem. We summarize as follows:

- In the Bayesian model, the optimal algorithm is $\mathrm{OPT}_{F}=\operatorname{argmax}_{M \in \mathcal{M} \text { OL }} M(F)$.

Consider the following family of binary independent stationary distributions $\mathcal{F}^{\text {BIS }}$ for the Bayesian variant of the expert learning problem. For a distribution $F \in \mathcal{F}^{\text {BIS }}$, each expert $j$ 's reward in each round is a Bernoulli random variable with mean $f_{j}$. The class $\mathcal{F}^{\text {BIS }}$ is composed of all possible means $f_{j} \in[0,1]$. At each round $t$, the rewards are drawn independently from each other and from other rounds. Importantly the distribution of each expert's reward is identical across rounds. For binary independent stationary distributions $F \in \mathcal{F}^{\mathrm{BIS}}$, the optimal algorithm picks the expert with the highest ex ante probability $j^{*}=\operatorname{argmax}_{j} f_{j}$ and follows expert $j^{*}$ in each round; its expected performance is

$$
\operatorname{OPT}_{F}(F)=n \max _{j} f_{j} .
$$

- In the prior-free model, the optimal algorithm is the one that minimizes regret in worst-case over inputs $\boldsymbol{v} \in \mathcal{V}$ against a given benchmark $B$ defined as

$$
\rho^{B}=\min _{M \in \mathcal{M}^{\mathrm{OL}}} \max _{\boldsymbol{v} \in \mathcal{V}}[B(\boldsymbol{v})-M(\boldsymbol{v})] .
$$

The best-in-hindsight benchmark for any reward profile $\boldsymbol{v} \in \mathcal{V}$ is

$$
B^{\mathrm{BIH}}(\boldsymbol{v})=\max _{j=1}^{k} \sum_{t=1}^{n} v_{t, j} .
$$

A typical online analysis measures performance in terms of worst-case regret with respect to the best-in-hindsight benchmark $B^{\mathrm{BIH}}$.

- In the prior-independent model, the optimal algorithm is the one that minimizes regret in worst-case over distributions $F \in \mathcal{F}$ against the optimal algorithm for the distribution

$$
\beta=\min _{M \in \mathcal{M}^{\mathrm{OL}}} \max _{F \in \mathcal{F}}\left[\operatorname{OPT}_{F}(F)-M(F)\right] .
$$

We will be considering this question for binary independent stationary distributions $\mathcal{F}^{\text {BIS }}$ where $\operatorname{OPT}_{F}(F)$ is as described above.

We observe next that the best-in-hindsight benchmark is normalized. (In fact, it is analogous to the normalized benchmark described by Hartline and Roughgarden (2008) for evaluating prior-free mechanisms.) Thus, an algorithm that is a prior-free approximation of the benchmark is also a prior-independent approximation algorithm (with the same bound on regret, cf. Proposition 1).

Lemma 14. For inputs from binary independent stationary distributions $\mathcal{F}^{\mathrm{BIS}}$, the best-in-hindsight benchmark $B^{\mathrm{BIH}}$ is normalized, i.e., $B^{\mathrm{BIH}}(F) \geq \operatorname{OPT}_{F}(F), \forall F \in \mathcal{F}^{\mathrm{BIS}}$.

Proof. Given $F \in \mathcal{F}^{\mathrm{BIS}}$, the Bayesian optimal algorithm $\mathrm{OPT}_{F}$ selects the same expert in each round. The best-in-hindsight benchmark selects the single expert that is best for the realized input $\boldsymbol{v}$. Thus, for all $\boldsymbol{v} \in \mathcal{V}, B^{\mathrm{BIH}}(\boldsymbol{v}) \geq \mathrm{OPT}_{F}(\boldsymbol{v})$. Taking expectations we have the lemma. 
We now show that the natural follow-the-leader algorithm, which in round $t$ chooses a uniform random expert from the set of experts with highest total reward from the first $t-1$ rounds, is the prior-independent optimal algorithm.

Definition 13. The follow-the-leader algorithm selects an expert uniformly at random from the set of experts with highest total reward from previous rounds:

$$
M_{t}^{\mathrm{FTL}}(\boldsymbol{v})=U\left[\operatorname{argmax}_{j} \sum_{t^{\prime}<t} v_{t^{\prime}, j}\right] .
$$

Theorem 9. For binary independent stationary distributions, the follow-the-leader algorithm is the prior-independent optimal online learning algorithm.

Proof. Consider the Bayesian optimal online algorithm for the uniform permutation prior defined by probabilities $\left\{f^{j}\right\}_{j=1}^{n}$ that are assigned to experts via a uniform random permutation $\sigma$ (i.e., the reward of expert $j$ is Bernoulli with mean $\left.f_{j}=f^{\sigma(j)}\right)$. The theorem follows from the optimality of follow-the-leader for any uniform permutation prior.

We first argue that the follow-the-leader algorithm is optimal for the any uniform permutation prior. The optimal algorithm for the uniform permutation prior forms a posterior from the reward history at any time $t$ and chooses the expert with the highest expectation under this posterior. Naturally, the experts with the highest expected reward under the posterior are the ones with the highest historical reward (formally, Lemma 17 in Appendix C). In other words, follow-the-leader is the Bayesian optimal algorithm for the uniform permutation prior.

As before denote by $\beta$ the prior-independent optimal regret. To complete the proof, consider the probabilities $\left\{f^{j}\right\}_{j=1}^{n}$ for which the Bayesian optimal algorithm for the uniform permutation prior obtains the largest regret. Observe that the regret of the Bayesian optimal algorithm for this uniform permutation prior lower bounds $\beta$. On the other hand, the prior-independent regret of the follow-the-leader algorithm upper bounds $\beta$. The follow-the-leader algorithm obtains the same regret on all permutations and this regret equals the Bayesian optimal regret for the uniform permutation prior; i.e., the upper bound and the lower bound are equal.

As we have proved in Section 2, benchmark optimization $(\sqrt{\gamma})$ and prior-independent optimization $(\beta)$ are the same. Thus, the optimal prior-free benchmark is the performance of the follow-theleader algorithm scaled up by its prior-independent approximation factor. Moreover, the optimal mechanism for the optimal benchmark is the follow-the-leader algorithm itself. While we may have hoped for the prior-free analysis to lead to more robust algorithms than the prior-independent analysis, by optimizing benchmarks in the framework provided in Section 2, we have lost all of this potential robustness. Specifically, the standard expert learning algorithms that have low worst-case regret against the best-in-hindsight benchmark exhibit robustness that the follow-the-leader lacks. This observation is formalized in the following lemma which contrasts with the optimal regret of standard algorithms like randomized weighted-majority (Littlestone and Warmuth, 1994). The optimal worst-case regret against best-in-hindsight is $\Theta(\sqrt{n \ln k})$ for $k$ experts, $n$ rounds, and binary rewards (Haussler et al., 1995).

Lemma 15. The prior-free regret of follow-the-leader against the best-in-hindsight benchmark with $n$ rounds is $\Theta(n)$.

Proof. Consider the input with an even number of rounds and rounds alternating as: 
- odd round payoffs: $(1,0, \ldots, 0) \in\{0,1\}^{k}$,

- even round payoffs: $(0,1, \ldots, 1) \in\{0,1\}^{k}$.

The follow-the-leader algorithm chooses a uniform random expert for odd rounds and obtains expected payoff $\frac{1}{k}$ and chooses expert 1 for even rounds and obtains expected payoff of 0 . The total expected payoff of follow the leader is $M^{\mathrm{FTL}}(\boldsymbol{v})=\frac{n}{2 k}$. On the other hand, the best-in-hindsight benchmark is $B^{\mathrm{BIH}}(\boldsymbol{v})=\frac{n}{2}$. The additive regret is $B^{\mathrm{BIH}}(\boldsymbol{v})-M^{\mathrm{FTL}}(\boldsymbol{v})=\frac{n}{2}\left(1-\frac{1}{k}\right) \in \Theta(n)$.

The observations of this section suggest that further study of the formulation of the benchmark optimization problem is necessary to better understand the trade-offs between prior-free and priorindependent robustness.

\section{Historical Context: Online Algorithms and Mechanism Design}

This section describes the historical development of online algorithms and mechanism design and compares approaches across these fields.

Online algorithms have been analyzed via a worst-case competitive analysis since Sleator and Tarjan (1985) with textbooks on the subject, e.g., Borodin and El-Yaniv (2005). In competitive analysis, the performance of an online algorithm is measured as its worst case ratio to the optimal offline algorithm. Good online algorithms are known with respect to this measure for many problems. For some problems this measure is too pessimistic, occurring when no good ratio is achievable by any algorithm and therefore good algorithms are not meaningfully separated from bad algorithms. The two approaches for resolving this issue are to either (a) restrict the offline algorithm to which the performance of the online algorithm is compared or (b) restrict the family of inputs that are considered. For this paper, the most relevant example of (a) comes from online learning where a learning algorithm's regret is measured with respect to the best fixed action in hindsight, i.e., to the optimal offline algorithm that is restricted to choose the same action in each time period (Littlestone and Warmuth, 1994; Freund and Schapire, 1997). The most relevant example of (b) for this paper is the diffuse adversary model of Koutsoupias and Papadimitriou (2000) which evaluates an algorithm as the ratio between its expected performance and the optimal offline performance in worst-case over a family of distributions on inputs.

Less immediately related to our work is a systematic study of analysis frameworks for online algorithms conducted by Boyar et al. (2015). They focus on a canonical two server problem and show how specific analysis frameworks result in different rankings of standard algorithms for the problem. These frameworks tend to vary the inputs compared between the algorithm and the benchmark rather than varying the benchmark itself (as we do). For example, the competitive ratio is the worst case over inputs of the optimal offline performance to the online algorithm's performance; while the max-max ratio compares the worst optimal offline performance to the worst algorithm performance, with both worst-cases taken over normalized inputs. They also consider analyses that directly compare algorithms rather than comparing the algorithms indirectly via a benchmark.

To place in context the development of robust analyses of mechanisms it is helpful to consider the predominant method for the analysis of mechanisms in economics. In economics, the preferences of the agents are assumed to be drawn from a known distribution and the design framework asks for the mechanism that maximizes performance (and satisfies incentive constraints) in expectation 
over this prior distribution over preferences. This area is known as Bayesian mechanism design, e.g., see the survey by Hartline (2013).

Competitive analysis was introduced to the design of mechanisms by Goldberg et al. (2006). While in online algorithms, the information theoretic barrier to good performance is the lack of information that comes from the online arrival of the input, for dominant strategy incentive compatible mechanism design, the information theoretic barrier to good performance comes from the need to satisfy the incentive constraints of the agents, namely that truthtelling is an equilibrium. The equivalent of the optimal offline algorithm, namely the optimal performance without incentives, is rarely an interesting benchmark as no good ratio is achievable. Both approaches (a) and (b) described above for online algorithms have been taken. For an example of (a), and with parallels to online learning, Goldberg et al. (2006) compares mechanisms for selling a digital good to the optimal revenue from posting a single price to all agents (i.e., the price-posting-revenue benchmark discussed in the introduction). They and the subsequent literature developed the area of prior-free mechanism design. In this area, the performance of a mechanism is compared to a benchmark performance in worst-case over inputs.

Lower bounds are important in the study of good mechanisms. Goldberg et al. (2006) introduced the following approach for establishing a lower bound on the prior-free approximation of any mechanism to a given benchmark. The approach considers a distribution over inputs for which all (undominated) mechanisms perform the same. For the objective of revenue maximization this distribution is known as the equal revenue distribution and has cumulative distribution function $F(z)=1-1 / z$ on support $[1, \infty)$. An agent with value drawn from this distribution offered any price $p \geq 1$ accepts with probability $1 / p$ and yields expected revenue 1. Goldberg et al. (2006) show a lower bound on the prior-free approximation to a posted-price-revenue based benchmark of 2.42 in the limit with the number of agents going to infinity. For the special case of $n=3$ agents, the lower bound is 3.25; Hartline and McGrew (2005) proved that this lower bound in the $n=3$ case is indeed tight by giving a mechanism that achieved it. Chen et al. (2014) proved that the lower bound of 2.42 is tight with a non-constructive proof and, moreover, that the lower-bounding method gives a tight bound for a large family of benchmarks that, like the one of Goldberg et al. (2006), are constant in the value of the highest agent.

Hartline and Roughgarden (2008) revisited the choice of benchmark of Goldberg et al. (2006) and identified the normalization constraint as presented in Section 1. Recall, they observe that if a benchmark satisfies the normalization constraint then mechanisms that approximate the benchmark in worst case also approximate the Bayesian optimal mechanism for any distribution in the family (with no worse an approximation factor). This consequence is known as the prior-independent corollary of approximation of a normalized benchmark. A specific normalized benchmark that they give is defined by the supremum of the performance of the Bayesian optimal mechanisms for each distribution in the family. For expert learning, this benchmark is in fact the best-in-hindsight benchmark (see Section 5). For auction settings, Devanur et al. (2015) give a simpler normalized benchmark based on relaxing the incentive constraints to constraints of envy-freedom.

Here we outline the origins of prior-independent analysis and compare it to the diffuse adversary approach in online algorithms. The prior-independent corollary of prior-free approximation of the benchmarks of Hartline and Roughgarden (2008) motivated the consideration of relaxing the assumption of worst-case inputs in a similar fashion to approach (b) above. A key difference, however, between the diffuse adversary model for online algorithms (Koutsoupias and Papadimitriou, 2000) and the prior-independent model is that the diffuse adversary model compares algorithms 
against the optimal offline benchmark (which relaxes the information theoretic constraints of the online optimization problem) where as, in prior-independent mechanism design, mechanisms are compared to the optimal mechanism for the distribution (that satisfies the incentive constraints). The advantage of considering distributions in the diffuse adversary model is more in the spirit of smoothed analysis. Dhangwatnotai et al. (2015) considered prior-independent mechanism design as a first-order goal and since then it has been the subject of a flourishing area of research.

The prior-independent mechanism design framework gives a natural question of identifying the optimal mechanism. This question is framed by a restriction to a family of distributions, but is not subject to an ad hoc performance benchmark as is prior-free mechanism design. Previous literature has only identified optimal prior-independent mechanisms in environments that are special cases of the fully general problem. Hartline and Roughgarden (2014) gave the prior-independent optimal mechanism for revenue maximization in the sale of a single item to a single agent with value from a bounded support where the prior-independent optimal mechanism posts a randomized price. For revenue maximization in the sale of an item to one of two agents with values drawn from an i.i.d. regular distribution, Dhangwatnotai et al. (2015) show that the second price auction is a 2approximation. Fu et al. (2015) gave a randomized mechanism showing that this factor of 2 is not tight. Upper and lower bounds on this canonical problem were improved by Allouah and Besbes (2018) to be within $[1.80,1.95]$.A main result of our paper is to identify the prior-independent optimal mechanism for this environment with a factor of about 1.913 For this two agent problem with i.i.d. values from a distribution in the subset of regular distributions that further satisfy a monotone hazard rate condition, Allouah and Besbes (2018) show that the second-price auction is optimal. As we see now, the optimality of the second price auction results from constraints binding that are loose when the class of distributions includes Triangles.

Upper and lower bounds on this canonical problem were improved by Allouah and Besbes (2018) to be within $[1.80,1.95] .4$ A main result of ourthis paper is to identify the prior-independent optimal mechanism for this environment with a factor of about 1.91. For this two agent problem with i.i.d. values from a distribution in the subset of regular distributions that further satisfy a monotone hazard rate condition, Allouah and Besbes (2018) show that the second-price auction is optimal.

In the context above, this paper formalizes the problem of identifying the normalized benchmark that yields the tightest approximation by a mechanism. We show that this question is equivalent to solving the prior-independent mechanism design problem. Thus, from our prior-independent optimal mechanism, we identify the optimal normalized benchmark for 2-agent revenue maximization with values drawn i.i.d. from regular distributions. Moreover, in contrast to the positive main result of Chen et al. (2014), we show that relaxing the program to find the optimal benchmark to the simpler program of finding the normalized benchmark that minimizes the lower bound from Goldberg et al. (2006) (i.e., from the equal revenue distribution) is not tight. Specifically, the lower bound for the benchmark that is optimal for the relaxed benchmark optimization program is not tight.

\footnotetext{
${ }^{3}$ The Allouah and Besbes (2018) lower bound of 1.80 was proved under the same additional assumption of scale invariance as our lower bound of 1.91 .

${ }^{4}$ Their lower bound of 1.80 holds under the additional assumption of scale invariance.
} 


\section{References}

Alaei, S., Hartline, J., Niazadeh, R., Pountourakis, E., and Yuan, Y. (2018). Optimal auctions vs. anonymous pricing. Games and Economic Behavior.

Allouah, A. and Besbes, O. (2018). Prior-independent optimal auctions. In Proceedings of the 2018 ACM Conference on Economics and Computation, pages 503-503. ACM.

Borodin, A. and El-Yaniv, R. (2005). Online computation and competitive analysis. cambridge university press.

Boyar, J., Irani, S., and Larsen, K. S. (2015). A comparison of performance measures for online algorithms. Algorithmica, 72(4):969-994.

Bulow, J. and Roberts, J. (1989). The simple economics of optimal auctions. The Journal of Political Economy, 97:1060-90.

Chen, N., Gravin, N., and Lu, P. (2014). Optimal competitive auctions. In Proceedings of the 46th Annual ACM Symposium on Theory of Computing, pages 253-262, New York, NY, USA. ACM.

Devanur, N. R., Hartline, J. D., and Yan, Q. (2015). Envy freedom and prior-free mechanism design. Journal of Economic Theory, 156:103-143.

Dhangwatnotai, P., Roughgarden, T., and Yan, Q. (2015). Revenue maximization with a single sample. Games and Economic Behavior, 91:318-333.

Feng, Y. and Hartline, J. D. (2018). An end-to-end argument in mechanism design (priorindependent auctions for budgeted agents). In 2018 IEEE 59th Annual Symposium on Foundations of Computer Science (FOCS), pages 404-415. IEEE.

Freund, Y. and Schapire, R. E. (1997). A decision-theoretic generalization of on-line learning and an application to boosting. Journal of computer and system sciences, 55(1):119-139.

Fu, H., Immorlica, N., Lucier, B., and Strack, P. (2015). Randomization beats second price as a prior-independent auction. In Proceedings of the Sixteenth ACM Conference on Economics and Computation, pages 323-323. ACM.

Goldberg, A. and Hartline, J. (2003). Envy-free auctions for digital goods. In Proc. 4th ACM Conf. on Electronic Commerce, pages 29-35. ACM Press.

Goldberg, A. V., Hartline, J. D., Karlin, A., Saks, M., and Wright, A. (2006). Competitive auctions. Games and Economic Behavior, 55:242-269.

Hartline, J. (2013). Bayesian mechanism design. Foundations and Trends $R$ in Theoretical Computer Science, 8(3):143-263.

Hartline, J. and McGrew, R. (2005). From optimal limited to unlimited supply auctions. In Proceedings of the 7th ACM Conference on Electronic Commerce, pages 175-182.

Hartline, J. and Roughgarden, T. (2008). Optimal mechanism design and money burning. In Proc. 39th ACM Symp. on Theory of Computing, pages 75-84. 
Hartline, J. D., Kleinberg, R., and Malekian, A. (2015). Bayesian incentive compatibility via matchings. Games and Economic Behavior, 92:401-429.

Hartline, J. D. and Roughgarden, T. (2014). Optimal platform design. arXiv preprint arXiv:1412.8518.

Haussler, D., Kivinen, J., and Warmuth, M. K. (1995). Tight worst-case loss bounds for predicting with expert advice. In Vitányi, P., editor, Computational Learning Theory, pages 69-83, Berlin, Heidelberg. Springer Berlin Heidelberg.

Koutsoupias, E. and Papadimitriou, C. H. (2000). Beyond competitive analysis. SIAM Journal on Computing, 30(1):300-317.

Littlestone, N. and Warmuth, M. K. (1994). The weighted majority algorithm. Information and computation, 108(2):212-261.

Myerson, R. (1981). Optimal auction design. Mathematics of Operations Research, 6:58-73.

Ronen, A. (2001). On approximating optimal auctions. In Proceedings of the 3rd ACM conference on Electronic Commerce, pages 11-17. ACM.

Sleator, D. D. and Tarjan, R. E. (1985). Amortized efficiency of list update and paging rules. Communications of the ACM, 28(2):202-208.

\section{A Missing Proofs from Section 3}

To identify the prior-independent optimal mechanism for triangle distributions, we evaluate the ratio of revenues of markup mechanisms on triangle distributions to the optimal revenue. For distribution $\operatorname{Tri}_{\bar{q}}$ the optimal revenue is $2-\bar{q}$ (Lemma 4). The revenue for $r$-markup mechanism is calculated by Lemma 6. In this appendix, we drive the formula of Lemma 6 and show that it has bounded partial derivatives in both markup $r$ and monopoly quantile $\bar{q}$. We then describe the details of the hybrid numerical and analytical argument of Theorem 4. Finally we give the proof of continuity of the adversary's best response distribution to the probability the mechanism places on the second-price auction.

\section{A.1 Derivation and smoothness of Lemma 6}

Proof of Lemma 6. Denote the quantile corresponding to the price $r V_{\operatorname{Tri}_{\bar{q}}}(q)$ for markup $r>1$ as

$$
\hat{Q}(q, r)=Q_{\operatorname{Tri}_{\bar{q}}}\left(r V_{\operatorname{Tr}_{\bar{q}}}(q)\right)= \begin{cases}\frac{q}{r-q r+q} & \text { if } r V_{\operatorname{Tr}_{\bar{q}}}(q) \leq 1 / \bar{q} \\ 0 & \text { otherwise }\end{cases}
$$

When the quantile of the second highest agent is smaller than $\hat{Q}(\bar{q}, 1 / r)$, the price $r \cdot v_{(2)}$ is higher than the support of the valuation distribution. Therefore, the revenue of posting price $r \cdot v_{(2)}$ to 
the highest bidder is

$$
\begin{aligned}
M_{r}\left(\operatorname{Tri}_{\bar{q}}\right) & =2 r \int_{\hat{Q}(\bar{q}, 1 / r)}^{1} V_{\operatorname{Tri}_{\bar{q}}}(q) \hat{Q}(q, r) d q \\
& =2 r \int_{\hat{Q}(\bar{q}, 1 / r)}^{1} \frac{1-q}{1-\bar{q}} \cdot \frac{1}{r-q r+q} d q=\frac{2 r}{1-\bar{q}}\left[\frac{q}{r-1}+\frac{\ln (r-q r+q)}{(r-1)^{2}}\right]_{\frac{\bar{q}}{1 / r-\bar{q} / r+\bar{q}}}^{1} \\
& =\frac{2 r}{(1-\bar{q})(r-1)}\left(\frac{1-\bar{q}}{1-\bar{q}+\bar{q} r}-\frac{\ln \left(\frac{r}{1-\bar{q}+\bar{q} r}\right)}{r-1}\right)
\end{aligned}
$$

where the second equality holds just by the definition of the distribution.

Consider the revenue of $r$-markup mechanism on the triangle distribution $\operatorname{Tr}_{\bar{q}}$ as a function of $r \in(1, \infty)$ and $\bar{q} \in[0,1]$. The formula for this revenue is given by Lemma 6 . The following two claims show that the ratio of revenues has bounded partial derivative with respect to both $r \in(1, \infty)$ and $\bar{q} \in[0,1]$ and, thus, numerical evaluation of the revenue at selected parameters allows large regions of parameter space to be ruled out.

Claim 1. For any distribution $F$ and any constants $1 \leq r_{1} \leq r_{2}$, we have $M_{r_{1}}(F) \geq r_{1} / r_{2} M_{r_{2}}(F)$.

Claim 2. For any mechanism $M_{r}$ with $r \geq 1$, and any constants $0 \leq \bar{q}_{1} \leq \bar{q}_{2}<1$, we have $\left(1-\bar{q}_{2}\right) /\left(1-\bar{q}_{1}\right) M_{r}\left(\operatorname{Tri}_{\bar{q}_{2}}\right) \leq M_{r}\left(\operatorname{Tri}_{\bar{q}_{1}}\right) \leq 2\left(\bar{q}_{2}-\bar{q}_{1}\right)+M_{r}\left(\operatorname{Tr}_{\bar{q}_{2}}\right)$.

Proof of Claim 1. For any realized valuation profile, if the item is sold in mechanism $M_{r_{2}}$, then the item is sold in mechanism $M_{r_{1}}$ since the price posted to the highest agent is smaller in mechanism $M_{r_{1}}$. Moreover, when the item is sold in mechanism $M_{r_{1}}$, the payment from agent with highest value is at least $r_{1} / r_{2}$ fraction of the payment in mechanism $M_{r_{2}}$. Taking expectation over the valuation profiles, we have $M_{r_{1}}(F) \geq r_{1} / r_{2} \cdot M_{r_{2}}(F)$.

Proof of Claim Q . Consider $\hat{Q}(\cdot, \cdot)$ as defined in the proof of Lemma [6, above. By directly comparing the revenue from two distributions,

$$
\begin{aligned}
M_{r}\left(\operatorname{Tri}_{\bar{q}_{1}}\right) & =2 r \int_{\hat{Q}\left(\bar{q}_{1}, 1 / r\right)}^{1} V_{\operatorname{Tr}_{\bar{q}_{1}}}(q) \hat{Q}(q, r) d q \\
& \leq 2\left(-\hat{Q}\left(\bar{q}_{1}, 1 / r\right)+\hat{Q}\left(\bar{q}_{2}, 1 / r\right)\right)+2 r \int_{\hat{Q}\left(\bar{q}_{2}, 1 / r\right)}^{1} V_{\operatorname{Tr}_{\bar{q}_{1}}}(q) \hat{Q}(q, r) d q \\
& \leq 2\left(\bar{q}_{2}-\bar{q}_{1}\right)+2 r \int_{\hat{Q}\left(\bar{q}_{2}, 1 / r\right)}^{1} V_{\operatorname{Tri}_{\bar{q}_{2}}}(q) \hat{Q}(q, r) d q \\
& =2\left(\bar{q}_{2}-\bar{q}_{1}\right)+M_{r}\left(\operatorname{Tr}_{\bar{q}_{2}}\right) .
\end{aligned}
$$

The first equality holds because the quantile of $V_{\operatorname{Tr}_{\bar{q}_{1}}}(q) \cdot r$ is 0 for $q<\hat{Q}\left(\bar{q}_{1}, 1 / r\right)$. The first inequality holds because $r \cdot V_{\operatorname{Tri}_{\bar{q}}}(q) \hat{Q}(q, r) \leq 1$ for any quantile $q$. The second inequality holds because $V_{\operatorname{Tri}_{\bar{q}_{1}}}(q) \leq V_{\operatorname{Tri}_{\bar{q}_{2}}}(q)$ for $\bar{q}_{1} \leq \bar{q}_{2}$ and $q \geq \bar{q}_{2}$ by the definition of distributions $\operatorname{Tri}_{\bar{q}_{1}}$ and 
$\operatorname{Tri}_{\bar{q}_{2}}$, and $\hat{Q}\left(\bar{q}_{2}, 1 / r\right)-\hat{Q}\left(\bar{q}_{1}, 1 / r\right) \leq \bar{q}_{2}-\bar{q}_{1}$. Moreover, we have

$$
\begin{aligned}
M_{r}\left(\operatorname{Tri}_{\bar{q}_{1}}\right) & =2 r \int_{\hat{Q}\left(\bar{q}_{1}, 1 / r\right)}^{1} V_{\operatorname{Tri}_{\bar{q}_{1}}}(q) \hat{Q}(q, r) d q \\
& \geq 2 r \int_{\hat{Q}\left(\bar{q}_{2}, 1 / r\right)}^{1} V_{\operatorname{Tr}_{\bar{q}_{1}}}(q) \hat{Q}(q, r) d q \\
& \geq \frac{2 r\left(1-\bar{q}_{2}\right)}{1-\bar{q}_{1}} \int_{\hat{Q}\left(\bar{q}_{2}, 1 / r\right)}^{1} V_{\operatorname{Tri}_{\bar{q}_{2}}}(q) \hat{Q}(q, r) d q \\
& =\frac{1-\bar{q}_{2}}{1-\bar{q}_{1}} \cdot M_{r}\left(\operatorname{Tri}_{\bar{q}_{2}}\right)
\end{aligned}
$$

where the first inequality holds because $\bar{q}_{1} \leq \bar{q}_{2}$ and function $\hat{Q}(q, r)$ is increasing in $q$. The second inequality holds because $V_{\operatorname{Tri}_{\bar{q}_{1}}}(q) \geq\left(1-\bar{q}_{2}\right) /\left(1-\bar{q}_{1}\right) \cdot V_{\operatorname{Tr}_{\bar{q}_{2}}}(q)$.

\section{A.2 Numerical and Analytical Arguments of Theorem 4}

The proof of Theorem 4 is based on a hybrid numerical and analytical argument. We can numerically calculate the revenue of a mechanism $M_{r}$ on a distribution $\operatorname{Tri}_{\bar{q}}$ via Lemma 6 and then we can argue, via Claim 2 and Claim 1, that nearby mechanisms and distributions have similar revenue. This approach will both allow us to argue about the structure of the solution and to identify the mechanism $M_{\alpha^{*}, r^{*}}$ and distribution of the solution $\operatorname{Tri}_{\bar{q}^{*}}$. Our subsequent discussion gives the details of these hybrid arguments.

We first approximate $\bar{q}^{*}$ by showing that $\bar{q}^{*} \in[0.09310569,0.09310571]$. The parameters for this range are found by discretizing the space and finding the optimal choice of $\bar{q}^{*}$. Note that the optimal choice of $\bar{q}^{*}$ satisfies $M_{1}\left(\operatorname{Tri}_{\bar{q}^{*}}\right)=M_{r\left(\bar{q}^{*}\right)}\left(\operatorname{Tri}_{\bar{q}^{*}}\right)$. Therefore, it is sufficient for us to show that for any quantile $\bar{q} \notin[0.09310569,0.09310571]$, either $M_{1}\left(\operatorname{Tri}_{\bar{q}}\right)>M_{r(\bar{q})}\left(\operatorname{Tri}_{\bar{q}}\right)$ or $M_{1}\left(\operatorname{Tr}_{\bar{q}}\right)<M_{r(\bar{q})}\left(\operatorname{Tri}_{\bar{q}}\right)$.

First we show for any $\bar{q} \in[0,0.09310569], M_{1}\left(\operatorname{Tr}_{\bar{q}}\right)<M_{r(\bar{q})}\left(\operatorname{Tri}_{\bar{q}}\right)$. Here we discretize the space $[0,0.09310569]$ into $Q_{d}$ with precision $\epsilon=10^{-9}$. By numerically calculation using Lemma 6 , we have

$$
\min _{\bar{q} \in Q_{d}} M_{2.446946}\left(\operatorname{Tri}_{\bar{q}}\right)=M_{2.446946}\left(\operatorname{Tri}_{0.09310569}\right) \geq 1+10^{-8}
$$

and for any $\bar{q} \in[0,0.09310569]$, letting $\bar{q}_{d}$ be the largest quantile in $Q_{d}$ smaller than or equal to $\bar{q}$, the minimum revenue for mechanism $M_{2.446946}$ is

$$
M_{2.446946}\left(\operatorname{Tri}_{\bar{q}}\right) \geq \frac{1-\bar{q}_{d}-\epsilon}{1-\bar{q}_{d}} \cdot M_{2.446946}\left(\operatorname{Tri}_{\bar{q}_{d}}\right) \geq 1+8 \times 10^{-9}>M_{1}\left(\operatorname{Tr}_{\bar{q}}\right),
$$

where the first inequality holds by Claim 2 and the second inequality holds because $\bar{q}_{d} \leq 0.1$.

Then we show for any $\bar{q} \in[0.09310571,1], M_{1}\left(\operatorname{Tri}_{\bar{q}}\right)>M_{r(\bar{q})}\left(\operatorname{Tri}_{\bar{q}}\right)$. We discretize the space $[0.09310571,1]$ into $\hat{Q}_{d}$ with precision $\hat{\epsilon}=10^{-9}$. First note that $M_{r}\left(\operatorname{Tri}_{\bar{q}}\right)<1$ for any $\bar{q} \geq 0.093$ and $r \geq 11$, since the expected probability the highest type got allocated is less than $\frac{1}{2}$, and hence the expected virtual value for mechanism $M_{r}$ with distribution $\operatorname{Tr}_{\bar{q}}$ is less than 1 . By Lemma 4 , the revenue in this case is less than 1 . With bounded range for optimal ratio $r$, we discretize the space $(1,11]$ into $R_{d}$ with precision $\epsilon_{r}=10^{-9}$. By numerically calculation using Lemma 6, we have

$$
\max _{\bar{q} \in \hat{Q}_{d}, r \in R_{d}} M_{r}\left(\operatorname{Tri}_{\bar{q}}\right)=M_{2.446945061}\left(\operatorname{Tri}_{0.09310571}\right) \leq 1-3 \times 10^{-8}
$$


and for any $\bar{q} \in[0.09310571,1]$ and any $r \in(1,11]$, letting $\bar{q}_{d}$ be the largest quantile in $\hat{Q_{d}}$ smaller than or equal to $\bar{q}$ and $r_{d}$ be the smallest number in $R_{d}$ larger than or equal to $r$, the maximum revenue for distribution $\operatorname{Tri}_{\bar{q}}$ is

$$
\max _{r \in(1,11]} M_{r}\left(\operatorname{Tri}_{\bar{q}}\right) \leq \frac{r_{d}}{r_{d}-\epsilon_{r}} \cdot\left(2 \hat{\epsilon}+M_{r_{d}}\left(\operatorname{Tri}_{\bar{q}_{d}}\right)\right) \leq 1-10^{-8}<M_{1}\left(\operatorname{Tri}_{\bar{q}}\right)
$$

where the first inequality holds by Claim 1 and 2 , and the second inequality holds because $r_{d}>1$. Combining the numerical calculation, we have that $\bar{q}^{*} \approx 0.0931057$.

Note that both mechanism $M_{1}$ and $M_{r^{*}}$ are the best responses for distribution $\operatorname{Tri}_{\bar{q}^{*}}$, achieving revenue 1 , and hence the optimal prior independent approximation ratio is

$$
\beta=\frac{\operatorname{OPT}_{\operatorname{Tri}_{\bar{q}^{*}}}\left(\operatorname{Tri}_{\bar{q}^{*}}\right)}{M_{\alpha^{*}, r^{*}}\left(\operatorname{Tri}_{\bar{q}^{*}}\right)}=2-\bar{q}^{*} \approx 1.9068943 .
$$

Next we show that by choosing ratio $r^{*} \approx 2.4469452$ and probability $\alpha^{*} \approx 0.80564048$, the prior independent approximation ratio of mechanism $M_{\alpha^{*}, r^{*}}$ approximates $\beta$. Here we discretize the quantile space $[0,1]$ into $Q_{d}^{\prime}$ with precision $\epsilon^{\prime}=10^{-9}$, using the formula in Lemma 4 and Lemma 6, the triangle distribution that maximizes the approximation ratio for mechanism $M_{\alpha^{*}, r^{*}}$ is $\operatorname{Tri}_{0.093105694}$ with approximation ratio at most 1.9068943044 . For any $\bar{q} \in\left[0, \frac{1}{2}\right]$, letting $\bar{q}_{d}$ be the largest quantile in $Q_{d}^{\prime}$ smaller than or equal to $\bar{q}$, the minimum revenue for mechanism $M_{\alpha^{*}, r^{*}}$ is

$M_{\alpha^{*}, r^{*}}\left(\operatorname{Tri}_{\bar{q}}\right) \geq \frac{1-\bar{q}_{d}-\epsilon^{\prime}}{1-\bar{q}_{d}} \cdot M_{\alpha^{*}, r^{*}}\left(\operatorname{Tri}_{\bar{q}_{d}}\right) \geq \frac{1}{1.906894309} \operatorname{OPT}_{\bar{q}_{d}}\left(\operatorname{Tri}_{\bar{q}_{d}}\right) \geq \frac{1}{1.906894309} \operatorname{OPT}_{\bar{q}}\left(\operatorname{Tri}_{\bar{q}}\right)$,

where the second inequality holds because $\bar{q}_{d} \leq \frac{1}{2}$ and the last inequality holds because $\bar{q}_{d} \leq \bar{q}$. For any $\bar{q} \in\left[\frac{1}{2}, 1\right]$, the minimum revenue for mechanism $M_{\alpha^{*}, r^{*}}$ is

$$
M_{\alpha^{*}, r^{*}}\left(\operatorname{Tri}_{\bar{q}}\right) \geq \alpha^{*} \cdot M_{1}\left(\operatorname{Tri}_{\bar{q}}\right) \geq 0.8 \geq \frac{1}{1.875} \operatorname{OPT}_{\bar{q}}\left(\operatorname{Tri}_{\bar{q}}\right)
$$

since for any $\bar{q} \in\left[\frac{1}{2}, 1\right], M_{1}\left(\operatorname{Tri}_{\bar{q}_{d}}\right)=1$ and $\operatorname{OPT}_{\bar{q}}\left(\operatorname{Tri}_{\bar{q}}\right)=2-\bar{q} \leq 1.5$. Therefore, $r^{*} \approx 2.4469452$ and probability $\alpha^{*} \approx 0.80564048$ are the desirable parameters, with error at most $2 \times 10^{-8}$ in prior independent approximation ratio. By our characterization, the error solely comes from numerical calculation, finishing the numerical analysis for Theorem 4 .

\section{A.3 Continuity of Distribution in Probability of Second-price Auction}

Recall the function $\bar{q}_{r}(\alpha)$ which gives the adversary's best-response triangle distribution the mechanism $M_{\alpha, r}$. The continuity of the function $\bar{q}_{r}(\alpha)$ is used to prove the existence of equilibrium between the randomized markup mechanism and the triangle distribution in Theorem 4 . The following claim proves the continuity of the function $\bar{q}_{r}(\alpha)$, by numerically bounding the second derivative of the revenue ratio of the stochastic markup mechanism $M_{\alpha, r}$ on distribution $\operatorname{Tri}_{\bar{q}}$ with respect to $\alpha$, the probability that the markup mechanism runs the second-price auction.

Claim 3. Given any $r \in[2.445,2.449]$, function $\bar{q}_{r}(\alpha)$ is continuous in $\alpha$ for $\alpha \in[0.8,0.81]$. 
Proof of Claim [3. By Lemma 6] and Theorem 2, the approximation ratio of mechanism $M_{\alpha, r}$ for triangle distribution $\operatorname{Tri}_{\bar{q}}$ is

$$
\begin{aligned}
\operatorname{APX}(\alpha, r, \bar{q}) & =\frac{\operatorname{OPT}_{\operatorname{Tr}_{\bar{q}}}\left(\operatorname{Tri}_{\bar{q}}\right)}{\alpha \cdot M_{1}\left(\operatorname{Tri}_{\bar{q}}\right)+(1-\alpha) M_{r}\left(\operatorname{Tri}_{\bar{q}}\right)} \\
= & \frac{2-\bar{q}}{\alpha+\frac{2 r(1-\alpha)}{(1-\bar{q})(r-1)}\left(\frac{1-\bar{q}}{1-\bar{q}+\bar{q} r}+\frac{\ln \left(\frac{r}{1-\bar{q}+\bar{q} r}\right)}{1-r}\right)}
\end{aligned}
$$

The approximation ratio is a continuous function of $\alpha, \bar{q}$. Therefore, to show that fixing $r$, function $\bar{q}_{r}(\alpha)$ is continuous in $\alpha$, it is sufficient to show that there is a unique $\bar{q}$ that maximizes $\operatorname{APX}(\alpha, r, \bar{q})$ for $r \in[2.445,2.449]$ and $\alpha \in[0.8,0.81]$, or equivalently, we show that there is a unique $\bar{q}$ that minimizes $1 / \operatorname{APX}(\alpha, r, \bar{q})$. By Claim 1 and 2 , we can discretize the quantile space and numerically verify that distributions with monopoly quantiles $\bar{q} \notin[0.093,0.094]$ are suboptimal. Therefore, we prove the uniqueness of the maximizer by showing that the second order derivative of $1 / \operatorname{APX}(\alpha, r, \bar{q})$ is strictly positive for $\bar{q} \in[0.093,0.094]$.

$$
\begin{aligned}
\frac{\partial^{2} \frac{1}{\operatorname{APX}(\alpha, r, \bar{q})}}{(\partial \bar{q})^{2}}= & \frac{4(1-\alpha) r\left(-\frac{r-1}{(1-\bar{q}+\bar{q} r)^{2}}+\frac{1}{(1-\bar{q})(1-\bar{q}+\bar{q} r)}-\frac{\log \left(\frac{r}{1-\bar{q}+\bar{q} r}\right)}{(r-1)(1-\bar{q})^{2}}\right)}{(r-1)(2-\bar{q})^{2}} \\
& +\frac{2(1-\alpha) r\left(-\frac{(r-1)^{2}}{(1-\bar{q}+\bar{q} r)^{3}}-\frac{r-1}{(1-\bar{q})(1-\bar{q}+\bar{q} r)^{2}}+\frac{2}{(1-\bar{q})^{2}(1-\bar{q}+\bar{q} r)}+\frac{2 \log \left(\frac{r}{1-\bar{q}+\bar{q} r}\right)}{(r-1)(1-\bar{q})^{3}}\right)}{(r-1)(2-\bar{q})} \\
& +\frac{4(1-\alpha) r\left(-\frac{1}{1-\bar{q}+\bar{q} r}-\frac{\log \left(\frac{r}{1-\bar{q}+\bar{q} r}\right)}{(r-1)(1-\bar{q})}\right)+2 \alpha(r-1)}{(r-1)(2-\bar{q})^{3}}
\end{aligned}
$$

By substituting the upper and lower bounds of $\alpha, r, \bar{q}$, we know that

$$
\frac{\partial^{2} \frac{1}{\operatorname{APX}(\alpha, r, \bar{q})}}{(\partial \bar{q})^{2}}>0.7
$$

for $r \in[2.445,2.449], \alpha \in[0.8,0.81]$ and $\bar{q} \in[0.093,0.094]$, which concludes the uniqueness of the maximizer and the continuity of function $\bar{q}_{r}(\alpha)$.

\section{B Missing Proof from Section 4}

We first establish the proofs of Lemma 12 and Lemma 13. We then give the proof of Theorem 8 and auxiliary lemmas that establish a gap between the relaxed benchmark design program and the benchmark design program for regular distributions.

Proof of Lemma 12 and Lemma 13. The proof for these two lemmas is similar to the numerical calculation in Theorem 4. For Lemma 12, we discretize the quantile space [0.05, 0.25] into $Q_{d}$ with precision $\epsilon=10^{-5}$, and compute the minimum approximation ratio of mechanism $M_{1.1}$ for any $\bar{q}$ 
in the discretized points in $Q_{d}$ using Lemma 6 (Section 3). By numerical calculation, the minimum approximation ratio is

$$
\min _{\bar{q} \in Q_{d}} \frac{\operatorname{OPT}_{\operatorname{Tr}_{\bar{q}}}\left(\operatorname{Tri}_{\bar{q}}\right)}{M_{1.1}\left(\operatorname{Tri}_{\bar{q}}\right)}=\frac{\mathrm{OPT}_{\operatorname{Tri}_{0.05}}\left(\operatorname{Tri}_{0.05}\right)}{M_{1.1}\left(\operatorname{Tri}_{0.05}\right)} \geq 2.0026
$$

and the minimum revenue for mechanism $M_{1.1}$ is

$$
\min _{q \in Q_{d}} M_{1.1}\left(\operatorname{Tri}_{\bar{q}}\right)=M_{1.1}\left(\operatorname{Tri}_{0.25}\right) \geq 0.74 \text {. }
$$

For any $\bar{q} \in[0.05,0.25]$, let $\bar{q}_{d}$ be the largest quantile in $Q_{d}$ smaller than or equal to $\bar{q}$, we have

$$
\begin{aligned}
& \frac{\mathrm{OPT}_{\operatorname{Tri}_{\bar{q}}}\left(\operatorname{Tri}_{\bar{q}}\right)}{M_{1.1}\left(\operatorname{Tr}_{\bar{q}}\right)} \geq \frac{\mathrm{OPT}_{\operatorname{Tr} \overline{\mathrm{q}}_{d}+\epsilon}\left(\operatorname{Tri}_{\bar{q}_{d}+\epsilon}\right)}{2 \epsilon+M_{1.1}\left(\operatorname{Tr}_{\bar{q}_{d}}\right)}=\frac{2-\bar{q}_{d}-\epsilon}{2-\bar{q}_{d}} \cdot \frac{\mathrm{OPT}_{\operatorname{Tri}_{\bar{q}}}\left(\operatorname{Tri}_{\bar{q}_{d}}\right)}{2 \epsilon+M_{1.1}\left(\operatorname{Tr}_{\bar{q}_{d}}\right)} \\
& \geq \frac{2-\bar{q}_{d}-\epsilon}{\left(2-\bar{q}_{d}\right)(1+3 \epsilon)} \cdot \frac{\mathrm{OPT}_{\operatorname{Tri}_{\bar{q}_{d}}}\left(\operatorname{Tri}_{\bar{q}_{d}}\right)}{M_{1.1}\left(\operatorname{Tri}_{\bar{q}_{d}}\right)} \geq 2,
\end{aligned}
$$

where the first inequality holds by applying Lemma 4 (Section 3 ) and Claim 2 (Appendix $\mathbb{A}$ ), and the equality holds by applying Lemma 4. The second inequality holds by noticing that $3 M_{1.1}\left(\operatorname{Tri}_{\bar{q}_{d}}\right) \geq$ $3 \times 0.74 \geq 2$.

We apply the similar analysis to the proof of Lemma 13. We discretize the quantile space $[0,0.05] \cup[0.25,1]$ into $\hat{Q}_{d}$ with precision $\hat{\epsilon}=10^{-5}$, and compute the minimum approximation ratio of mechanism $M_{\alpha^{*}, r^{*}}$ for any $\bar{q}$ in the discretized points in $\hat{Q}_{d}$ using Lemma 6. By numerical calculation, the minimum approximation ratio in $\hat{Q}_{d}$ is

$$
\min _{\bar{q} \in \hat{Q}_{d}} \frac{\operatorname{OPT}_{\operatorname{Tri}_{\bar{q}}}\left(\operatorname{Tri}_{\bar{q}}\right)}{M_{\alpha^{*}, r^{*}}\left(\operatorname{Tri}_{\bar{q}}\right)}=\frac{\operatorname{OPT}_{\operatorname{Tri}_{0.05}}\left(\operatorname{Tri}_{0.05}\right)}{M_{\alpha^{*}, r^{*}}\left(\operatorname{Tri}_{0.05}\right)} \leq 1.90406
$$

and for any $\bar{q} \in[0,0.05] \cup[0.25,0.5]$, we have

$$
\frac{\operatorname{OPT}_{\operatorname{Tri}}\left(\operatorname{Tri}_{\bar{q}}\right)}{M_{\alpha^{*}, r^{*}}\left(\operatorname{Tr}_{\bar{q}}\right)} \leq \frac{1-\bar{q}_{d}}{1-\bar{q}_{d}-\epsilon} \cdot \frac{\operatorname{OPT}_{\operatorname{Tri}_{\bar{q}_{d}}}\left(\operatorname{Tr}_{\bar{q}_{d}}\right)}{M_{\alpha^{*}, r^{*}}\left(\operatorname{Tri}_{\bar{q}_{d}}\right)} \leq 1.9041,
$$

where the first inequality holds by applying Lemma 4 and Claim 2 and the last inequality holds because $\bar{q} \leq 0.5$. For any $\bar{q} \in[0.5,1]$, we have

$$
\frac{\operatorname{OPT}_{\operatorname{Tri}_{\bar{q}}}\left(\operatorname{Tri}_{\bar{q}}\right)}{M_{\alpha^{*}, r^{*}}\left(\operatorname{Tri}_{\bar{q}}\right)} \leq \frac{\operatorname{OPT}_{\operatorname{Tri}_{0.5}}\left(\operatorname{Tri}_{0.5}\right)}{\alpha^{*} \cdot M_{1}\left(\operatorname{Tr}_{\bar{q}}\right)} \leq 1.88,
$$

and combining the inequalities gives the desired result.

The main difference for proving the gap for distributions comparing to triangle distributions is that the space for regular distributions is larger and we need to define more carefully what it means to have a distribution close to the worst case triangle distribution $\operatorname{Tri}_{\bar{q}}$. By Theorem 3 , let $M_{\alpha^{*}, r^{*}}$ be the approximately optimal prior-independent mechanism with numerical parameters $\alpha^{*}=0.80564048, r^{*}=2.4469452$. We first define a pseudo-mechanism $\tilde{M}$ as the affine combination of mechanisms with revenue $\tilde{M}(\boldsymbol{v})=(1+\delta) M_{\alpha^{*}, r^{*}}(\boldsymbol{v})-\delta \cdot M_{1.18}(\boldsymbol{v})$, where $\delta>0$ is a small constant. Recall that $\mathcal{F}^{\text {Reg }}$ is the family of regular distributions. We show that 


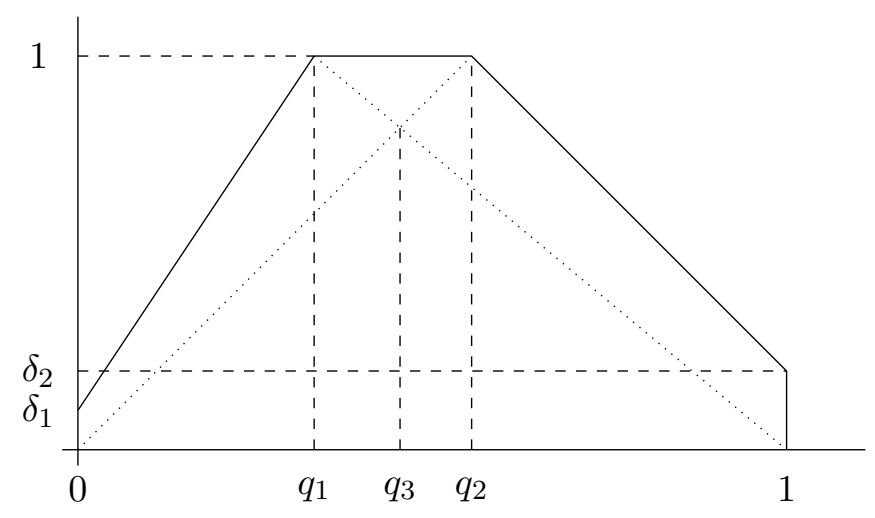

Figure 8: The revenue curve for distribution $\bar{F}$.

1. $\forall F \in \mathcal{F}^{\text {Reg }}, \tilde{M}(F) \leq \operatorname{OPT}_{F}(F)$;

2. $\forall F \in \mathcal{F}^{\text {Reg }}, \beta^{\prime} \tilde{M}(F) \geq \operatorname{OPT}_{F}(F)$, where $\beta^{\prime}<\beta$.

Letting $B(\boldsymbol{v})=\beta^{\prime} \cdot \tilde{M}(\boldsymbol{v})$, benchmark $B$ is normalized for all regular distributions and the approximation of optimal payoff against benchmark $B$ is $\beta^{\prime}<\beta=\gamma$. Therefore, the approximation of the optimal heuristic benchmark is $\bar{\gamma} \leq \beta^{\prime}<\gamma$ and Theorem 8 holds.

The first statement holds by Lemma 11. To show the second statement holds, since we know the worst case distribution $\operatorname{Tri}_{q^{*}}$ for the optimal prior-independent mechanism $M_{\alpha^{*}, r^{*}}$, intuitively, it is sufficient to show that mechanism $M_{1.18}$ has lower revenue for any distribution close to distribution $\operatorname{Tri}_{q^{*}}$. Formally, for any $0<q_{1}<q_{2}<1,0<\delta_{1}, \delta_{2}<1$, let $\bar{F}$ be the distribution such that

$$
R_{\bar{F}}(q)= \begin{cases}\frac{q\left(1-\delta_{1}\right)}{q_{1}}+\delta_{1} & 0 \leq q \leq q_{1}, \\ 1 & q_{1}<q<q_{2}, \\ \frac{q\left(\delta_{2}-1\right)}{1-q_{2}}+\delta_{2}+\frac{1-\delta_{2}}{1-q_{2}} & q_{2} \leq q \leq 1 .\end{cases}
$$

The revenue curve for distribution $\bar{F}$ is shown in Figure 8 . Note that if distribution $F$ is close to distribution $\operatorname{Tri}_{q^{*}}$, then naturally we have $F$ stochastically dominated by distribution $\bar{F}$. Before proving Theorem 8 , we first propose a formula for upper bounding the revenue of $M_{r}$ for any distribution $F$ stochastically dominated by $\bar{F}$, which will be used later to upper bound the revenue of mechanism $M_{1.18}$ for any distribution close to $\operatorname{Tri}_{\bar{q}^{*}}$. According to Figure 8 , letting $q_{3}=q_{2} /\left(1-q_{1}+q_{2}\right)$ and $q_{4}=Q_{\bar{F}}\left(1 / q_{2}\right)=\frac{r q_{2}\left(1-q_{2} \delta_{2}\right)}{1-q_{2}+r q_{2}\left(1-\delta_{2}\right)}$, we upper bound the revenue of mechanism $M_{r}$ for distribution 
$F$ by

$$
\begin{aligned}
& \frac{1}{2} \cdot M_{r}(F)=\int_{0}^{1} \bar{R}_{F}\left(V_{F}(q) \cdot r\right) d q \\
= & \int_{0}^{q_{3}} \bar{R}_{F}\left(V_{F}(q) \cdot r\right) d q+\int_{q_{3}}^{\hat{Q}\left(q_{1}, 1 / r\right)} \bar{R}_{F}\left(V_{F}(q) \cdot r\right) d q \\
& +\int_{\hat{Q}\left(q_{1},{ }^{1 / r}\right)}^{q_{4}} \bar{R}_{F}\left(V_{F}(q) \cdot r\right) d q+\int_{q_{4}}^{1} \bar{R}_{F}\left(V_{F}(q) \cdot r\right) d q \\
\leq & \frac{r q_{1} q_{3} \delta_{1}}{q_{1} r-\left(1-\delta_{1}\right) q_{2}}+\int_{q_{3}}^{\hat{Q}\left(q_{1}, 1 / r\right)} \bar{R}_{F}\left(\frac{r(1-q)}{1-q_{1}}\right) d q+q_{4}-\frac{r q_{1}}{r-q_{1}+r q_{1}}+\int_{q_{4}}^{1} \bar{R}_{F}\left(V_{F}(q) \cdot r\right) d q \\
\leq & \frac{r q_{1} q_{3} \delta_{1}}{q_{1} r-\left(1-\delta_{1}\right) q_{2}}+\int_{q_{3}}^{\hat{Q}\left(q_{1}, 1 / r\right)} \frac{r q_{1} \delta_{1}(1-q)}{r q_{1}(1-q)-q\left(1-\delta_{1}\right)\left(1-q_{1}\right)} d q \\
& +q_{4}-\frac{r q_{1}}{r-q_{1}+r q_{1}}+\int_{q_{4}}^{1} \frac{1}{1-q_{2}}\left(1-q_{2} \delta_{2}-\frac{\left(1-q_{2} \delta_{2}\right)\left(1-\delta_{2}\right)}{-r\left(1-\delta_{2}\right)+r\left(1-q_{2} \delta_{2}\right) / q+\left(1-\delta_{2}\right)}\right) d q .
\end{aligned}
$$

The first inequality holds because for any $q \leq q_{3}$, the price $V_{F}(q) \cdot r$ is higher than $1 / q_{1}$ and the revenue curve has positive slope. Therefore, the revenue is higher if the price is lower, which is at least $1 / q_{2}$. Moreover, $\bar{R}_{F}\left(V_{F}(q) \cdot r\right) \leq 1$ for any quantile $q$. The second inequality holds similarly since the slope on revenue curve is negative for $q \geq q_{4}$. With this upper bound on the revenue, we can continue to the numerical calculation for the gap between two programs.

Proof of Theorem 8 . We prove the gap by upper bounding the revenue of mechanism $M_{1.18}$ and lower bounding the revenue of mechanism $M_{\alpha^{*}, r^{*}}$ for regular distributions parameterized by their monopoly quantile $\bar{q}$.

First we upper bounding the revenue of mechanism $M_{1.18}$. By substituting $r=1.18, q_{1}=$ $0.09, q_{2}=0.098, \delta_{1}=\delta_{2}=0.01$ as parameter for distribution $\bar{F}$, we have $M_{1.18}(F) \leq 0.98444$ for any distribution $F$ stochastically dominated by $\bar{F}$ by Inequality (15), and $M_{1.18}(F) \leq \mathrm{OPT}_{F}(F)$ otherwise.

Next we bound the revenue of mechanism $M_{\alpha^{*}, r^{*}}$ for different sets of regular distributions. If the distribution $F$ is stochastically dominated by $\bar{F}$, by numerical result in Appendix $\mathrm{A}$, the revenue of mechanism $M_{\alpha^{*}, r^{*}}$ is at least $1 / 1.90689431 \cdot \mathrm{OPT}_{F}(F)$. If distribution $F$ is not stochastically dominated by $\bar{F}$, we can divide the analysis for mechanism $M_{\alpha^{*}, r^{*}}$ into two cases.

1. When $\bar{q} \notin[0.0905,0.096]$. In this case, the approximation ratio of mechanism $M_{\alpha^{*}, r^{*}}$ for distribution $F$ is at most the approximation ratio for triangle distribution $\operatorname{Tri}_{\bar{q}}$, which is at least 1.9068845 by discretizing the quantile space and applying numerical computation using Lemma 6 and Claim [2, i.e., the revenue of mechanism $M_{\alpha^{*}, r^{*}}$ in this case is at least $0.5244156 \cdot \mathrm{OPT}_{F}(F)$.

2. When $\bar{q} \in[0.0905,0.096]$, since the distribution $F$ is not stochastically dominated by $\bar{F}$, distribution $F$ is not a triangle distribution. Here we consider two subcases.

(a) There exists $q>0.096$ such that $R_{F}(q)>R_{\bar{F}}$. In this case, the distribution $F$ maximizes the approximation ratio is the truncated distribution and the optimal revenue 
for distribution $F$ is $2-\bar{q}$. Since distribution $F$ is not a triangle distribution, and by Claim 3, the increase of revenue for mechanism $M_{\alpha^{*}, r^{*}}$ is at least 0.0005 , and thus

$$
M_{\alpha^{*}, r^{*}}(F) \geq \frac{1}{1.90689431} \cdot \operatorname{OPT}_{F}(F)+0.0005 \geq 0.5246 \mathrm{OPT}_{F}(F)
$$

where the last inequality holds because $\mathrm{OPT}_{F}(F) \leq 2$.

(b) There exists $q<0.0905$ such that $R_{F}(q)>R_{\bar{F}}$. In this case, the revenue of the optimal mechanism for distribution $F$ increases by at least 0.0009 comparing to triangle distribution $\operatorname{Tr}_{\bar{q}}$, and the second price auction increases by at least this much. Therefore,

$$
\frac{M_{\alpha^{*}, r^{*}}(F)}{\mathrm{OPT}_{F}(F)} \geq \frac{M_{\alpha^{*}, r^{*}}\left(\operatorname{Tri}_{\bar{q}}\right)+0.0009 \alpha^{*}}{\mathrm{OPT}_{\operatorname{Tr} \bar{i}_{\bar{q}}}\left(\operatorname{Tri}_{\bar{q}}\right)+0.0009} \geq 0.5245
$$

where the last inequality holds because $M_{\alpha^{*}, r^{*}}\left(\operatorname{Tri}_{\bar{q}}\right) \geq 0.5244156 \cdot \operatorname{OPT}_{F}\left(\operatorname{Tri}_{\bar{q}}\right)$, and $\mathrm{OPT}_{\operatorname{Tri} \bar{q}}\left(\operatorname{Tri}_{\bar{q}}\right) \geq 2-\bar{q} \geq 1.905$ for $\bar{q} \leq 0.095$.

Combining the two subcases, we have that the revenue of mechanism $M_{\alpha^{*}, r^{*}}$ is at least $0.5245 \cdot \operatorname{OPT}_{F}(F)$ for $F$ not dominated by $\bar{F}$ and $\bar{q} \in[0.091,0.095]$.

By setting $\delta=5.21 \times 10^{-6}$, for both cases, we have $\beta^{\prime} \leq 1.90689356 \leq \beta-7 \times 10^{-7}$, where the last inequality holds because as we illustrated in the numerical result in Appendix $\mathrm{A}$, the optimal prior independent approximation ratio is $\beta=2-\bar{q}^{*} \geq 2-0.09310571=1.90689429$. By setting the benchmark $B(\boldsymbol{v})=1.90689422\left[(1+\delta) M_{\alpha^{*}, r^{*}}(\boldsymbol{v})-\delta \cdot M_{1.18}(\boldsymbol{v})\right]$, benchmark $B$ is normalized and the approximation ratio of the optimal revenue against benchmark $B$ is at most 1.90689422 . Therefore, the approximation of optimal revenue against the optimal heuristic benchmark is at most the approximation against benchmark $B$, which implies $\bar{\gamma} \leq \beta^{\prime}<\beta=\gamma$.

\section{Missing Proof from Section 5}

Recall from the proof of Theorem 9 that permutation distributions are defined by probabilities $\left\{f^{j}\right\}_{j=1}^{n}$ assigned to experts via a uniform random permutation $\sigma$ (the reward of expert $j$ is Bernoulli with mean $\left.f_{j}=f^{\sigma(j)}\right)$. Here we formally prove that, for permutation distributions, follow-the-leader $M^{\mathrm{FTL}}$ chooses the optimal expert according to the posterior distribution induced by the realized reward history.

At any time $t$, let the cumulative reward $V_{t, j}=\sum_{t^{\prime}<t} v_{t^{\prime}, j}$ be the total number of 1 's realized for expert $j$ until time $t-1$ and let

$$
j_{t}^{c}=\operatorname{argmax}_{j} V_{t, j}
$$

be the best expert chosen by $M^{\mathrm{FTL}}$. Let

$$
j_{t}^{p}=\operatorname{argmax}_{j} \mathbf{E}\left[v_{t, j} \mid\left\{v_{t^{\prime}}\right\}_{t^{\prime}<t}\right]
$$

be expert that maximizes the expected reward for the posterior distribution. Hartline et al. (2015) show that when there are two experts, the expert $j_{t}^{p}$ that maximizes the expected payoff according

to the posterior is the expert $j_{t}^{c}$ that maximizes the cumulative reward $V_{t, j}$. That is, $j_{t}^{p}=j_{t}^{c}$ for any time $t$. In Lemma 17, we generalize the result to $m$ experts for any $m \geq 2$. 
Definition 14. Let $f_{1} \geq f_{2} \geq \cdots \geq f_{m}$ be a sequence of probabilities for binary independent stationary distributions $F$. Distribution $F$ is non-degenerate if $f_{1}>f_{m}$ and there exists $k$ such that $f_{k} \in(0,1)$.

We prove the results in this section for non-degenerate distributions, and the degenerate case is trivial.

Lemma 16 (Lemma 4.3 in Hartline et al., 2015). At any time $t$, when there are two experts 1,2 with cumulative reward $V_{t, 1}>V_{t, 2}$ and non-degenerate distribution with probability $f_{1}>f_{2}$,

$$
\operatorname{Pr}\left[\sigma(1)=1 \text { and } \sigma(2)=2 \mid\left\{v_{t^{\prime}}\right\}_{t^{\prime}<t}\right]>\operatorname{Pr}\left[\sigma(1)=2 \text { and } \sigma(2)=1 \mid\left\{v_{t^{\prime}}\right\}_{t^{\prime}<t}\right]
$$

the probabilities are equal if $V_{t, 1}=V_{t, 2}$ or $f_{1}=f_{2}$.

Proof. The proof can be found in Hartline et al. (2015), and we include it here for completeness. The lemma trivially holds if $f_{1}=1$ or $f_{2}=0$. Otherwise, we have $f_{1}, f_{2} \in(0,1)$ and

$$
\begin{aligned}
\frac{\operatorname{Pr}\left[\sigma(1)=1 \text { and } \sigma(2)=2 \mid\left\{v_{t^{\prime}}\right\}_{t^{\prime}<t}\right]}{\operatorname{Pr}\left[\sigma(1)=2 \text { and } \sigma(2)=1 \mid\left\{v_{t^{\prime}}\right\}_{t^{\prime}<t}\right]} & =\frac{f_{2}^{V_{t, 2}} \cdot\left(1-f_{2}\right)^{t-V_{t, 2}} \cdot f_{1}^{V_{t, 1}} \cdot\left(1-f_{1}\right)^{t-V_{t, 1}}}{f_{2}^{V_{t, 1}} \cdot\left(1-f_{2}\right)^{t-V_{t, 1}} \cdot f_{1}^{V_{t, 2}} \cdot\left(1-f_{1}\right)^{t-V_{t, 2}}} \\
& =\left(1+\frac{f_{1}-f_{2}}{f_{2}\left(1-f_{1}\right)}\right)^{V_{t, 1}-V_{t, 2}}
\end{aligned}
$$

which is strictly larger than 1 ; the inequality is an equality if $V_{t, 1}=V_{t, 2}$ or $f_{1}=f_{2}$.

Lemma 17. For any $m \geq 2$, any non-degenerate distribution $F$, any pair of experts $j, j^{\prime}$ and for any time $t, \mathbf{E}\left[v_{t, j} \mid\left\{v_{t^{\prime}}\right\}_{t^{\prime}<t}\right]>\mathbf{E}\left[v_{t, j^{\prime}} \mid\left\{v_{t^{\prime}}\right\}_{t^{\prime}<t}\right]$ if $V_{t, j}>V_{t, j^{\prime}}$, and the expectations are equal if $V_{t, j}=V_{t, j^{\prime}}$.

Proof. Let $f_{1} \geq f_{2} \geq \cdots \geq f_{m}$ be a sequence of probabilities for non-degenerate distribution $F$. Suppose there is a uniform random permutation $\sigma$ such that the reward distribution for expert $j$ is $f_{\sigma(j)}$. For any expert $j^{\prime}$ with $V_{t, j}>V_{t, j^{\prime}}$, the expected reward conditional on the reward from time 1 to $t-1$ is

$$
\begin{aligned}
\mathbf{E}\left[v_{t, j} \mid\left\{v_{t^{\prime}}\right\}_{t^{\prime}<t}\right] & =\sum_{s} f_{s} \operatorname{Pr}\left[\sigma(j)=s \mid\left\{v_{t^{\prime}}\right\}_{t^{\prime}<t}\right] \\
& =\sum_{s, s^{\prime}} f_{s} \operatorname{Pr}\left[\sigma(j)=s \text { and } \sigma\left(j^{\prime}\right)=s^{\prime} \mid\left\{v_{t^{\prime}}\right\}_{t^{\prime}<t}\right] \\
& >\sum_{s, s^{\prime}} f_{s} \operatorname{Pr}\left[\sigma\left(j^{\prime}\right)=s \text { and } \sigma(j)=s^{\prime} \mid\left\{v_{t^{\prime}}\right\}_{t^{\prime}<t}\right] \\
& =\sum_{s} f_{s} \operatorname{Pr}\left[\sigma\left(j^{\prime}\right)=s \mid\left\{v_{t^{\prime}}\right\}_{t^{\prime}<t}\right] \\
& =\mathbf{E}\left[v_{t, j^{\prime}} \mid\left\{v_{t^{\prime}}\right\}_{t^{\prime}<t}\right],
\end{aligned}
$$

where the inequality holds by Lemma 16, it becomes an equality if $V_{t, j}=V_{t, j^{\prime}}$.

From Lemma 17, we immediately have the following theorem.

Theorem 10. For the uniform permutation of any non-degenerate binary independent stationary distribution $F$, the follow-the-leader algorithm $M^{\mathrm{FTL}}$ is the strict optimal algorithm that maximizes the expected reward given the posterior distribution in each round $t$. 


\section{Gap for Expert Learning}

In this section, we show that the gap between program $(\bar{\gamma})$ and $(\bar{\gamma})$ exists for expert learning as well. First we make the following observation for follow-the-leader with non-degenerate distributions.

Lemma 18. For any non-degenerate binary independent stationary distribution $F$, the expected performance of follow-the-leader at any round $t$ is less than its expected performance at round $t+1$, i.e., $\mathbf{E}\left[M_{t}^{\mathrm{FTL}}(\boldsymbol{v})\right]<\mathbf{E}\left[M_{t+1}^{\mathrm{FTL}}(\boldsymbol{v})\right]$.

Proof. Consider the uniform permutation environment under any non-degenerate distribution $F$. Theorem 10 shows that follow-the-leader is strictly optimal in every round. Specifically, any algorithm that selects an expert that does not have the highest historical reward obtains strictly smaller expected reward in this round.

Now consider two algorithms for selecting an expert in round $t+1$ :

1. follow the leader from rounds $\{1, \ldots, t-1\}$, or

2. follow the leader from rounds $\{1, \ldots, t\}$.

The expected round $t+1$ payoffs of these algorithms are $\mathbf{E}\left[M_{t}^{\mathrm{FTL}}(\boldsymbol{v})\right]$ and $\mathbf{E}\left[M_{t+1}^{\mathrm{FTL}}(\boldsymbol{v})\right]$, respectively. Under the assumption that the distribution is non-degenerate, there is a positive probability that the sets of experts with the highest historical rewards before round $t$ and $t+1$ are distinct; thus, by Theorem 10 the payoff of the former is strictly less than the payoff of the latter.

Of course, the follow-the-leader algorithm obtains the same payoff regardless of the permutation of probabilities to experts; thus, the strict inequality of the lemma holds for any non-degenerate binary independent stationary distribution $F$.

Theorem 11. For binary independent stationary reward distributions in expert learning problem with time horizon $n \geq 2$, the heuristic benchmark optimization program ( $\bar{\gamma}$ ) has a strictly smaller objective value than the benchmark optimization program $(\bar{\gamma})$, i.e., $\bar{\gamma}<\gamma$.

Proof. For benchmark optimization program $(\sqrt[\gamma]{)})$, the optimal benchmark is scaled-up follow-theleader $\beta \cdot M^{\mathrm{FTL}}$, where $\beta$ is the prior-independent approximation ratio for follow-the-leader $M^{\mathrm{FTL}}$, and $\gamma=\beta$.

Now consider another benchmark $B(\boldsymbol{v})=\beta^{\prime} \tilde{M}(\boldsymbol{v})$, where $\tilde{M}(\boldsymbol{v})=n \cdot M_{n}^{\mathrm{FTL}}(\boldsymbol{v})$ and $\beta^{\prime}$ is the prior-independent approximation ratio for $\tilde{M}$. It is easy to verify that for any binary independent stationary $F$ with probabilities $f_{1}, \ldots, f_{k}$,

$$
\tilde{M}(F)=n \cdot \mathbf{E}\left[M_{n}^{\mathrm{FTL}}(\boldsymbol{v})\right] \leq n \cdot \max _{j} f_{j}=\mathrm{OPT}_{F}(F) .
$$

Therefore, benchmark $B$ is normalized and the resolution of benchmark $B$ with respect to program $(\sqrt{\gamma})$ is at most $\beta^{\prime}$. It is sufficient to prove $\beta^{\prime}<\beta$ to prove Theorem [11.

First we show that degenerate distributions are not worst case for prior-independent approximation for either follow-the-leader $M^{\mathrm{FTL}}$ or pseudo-mechanism $\tilde{M}$. Note that for any distribution such that the expected reward for all experts are the same, we have $M^{\mathrm{FTL}}(F)=\tilde{M}(F)=\mathrm{OPT}_{F}(F)$, and hence such a distribution is not the worst case. Moreover, for degenerate distribution $F$ such that the expected reward for experts are not all the same, we can regenerate a non-degenerate distribution $F^{\prime}$ such that the prior-independent approximation ratio for both follow-the-leader $M^{\mathrm{FTL}}$ 
and pseudo-mechanism $\tilde{M}$ are worse, by switching the distribution of receiving reward 1 with probability 0 , to the distribution of receiving reward 1 with probability $\frac{1}{2}$. This does not affect the expected performance of the optimal mechanism, but strictly decreases the expected performance of follow-the-leader $M^{\text {FTL }}$ and pseudo-mechanism $\tilde{M}$.

Now we focus on distribution $F$ that is non-degenerate. We show that for any such distribution, $M^{\mathrm{FTL}}(F)<\tilde{M}(F)$, which implies $\beta^{\prime}<\beta$. Formally, we have that

$$
M^{\mathrm{FTL}}(F)=\mathbf{E}_{\boldsymbol{v}}\left[\sum_{t \leq n} M_{t}^{\mathrm{FTL}}(\boldsymbol{v})\right]<\mathbf{E}_{\boldsymbol{v}}\left[\sum_{t \leq n} M_{n}^{\mathrm{FTL}}(\boldsymbol{v})\right]=\tilde{M}(F)
$$

where the inequality holds by Lemma 18 and $n \geq 2$. 